\title{
Ribosomal protein S6 kinase1 coordinates with TOR-Raptor2 to regulate thylakoid membrane biosynthesis in rice
}

\author{
Linxiao Sun, Yonghua Yu, Weiqin Hu, Qiming Min, Huiling Kang, Yilu Li, Yue Hong, \\ Xuemin Wang, Yueyun Hong*
}

National Key Laboratory of Crop Genetic Improvement, College of Life Science and Technology, Huazhong Agricultural University, Wuhan 430070, China

\section{One-sentence summary:}

TOR/Raptor2/S6K1 is involved in modulating thylakoid membrane lipid biogenesis and lipid homeostasis to enhance photosynthesis in rice.

\section{Author for correspondence:}

Yueyun Hong

Tel: $86-27-87280545$

Email: hongyy@mail.hzau.edu.cn

\begin{abstract}
Ribosomal protein S6 kinase (S6K) functions as a key component in the target of rapamycin (TOR) pathway involved in multiple processes in eukaryotes. The role and regulation of TOR-S6K in lipid metabolism remained unknown in plants. Here we provide genetic and pharmacological evidence that TOR-Raptor2-S6K1 is important for thylakoid galactolipid biosynthesis and thylakoid grana modeling in rice (Oryza sativa L.). Genetic suppression of S6K1 caused pale yellow-green leaves, defective thylakoid grana architecture. S6K1 directly interacts with Raptor2, a core component in TOR signaling, and S6K1 activity is regulated by Raptor2 and TOR. Plants with suppressed Raptor2 expression or reduced TOR activity by inhibitors mimicked the S6K1-deficient phenotype. A significant reduction in galactolipid content was found in the $s 6 k 1$, raptor 2 mutant or TOR-inhibited plants, which was accompanied by decreased transcript levels of the set of genes such as lipid phosphate phosphatase $\alpha 5$ (LPP $\alpha 5), M G D G$ synthase 1 (MGD1), and $D G D G$ synthase 1 (DGD1) involved in galactolipid synthesis, compared to the control
\end{abstract}


plants. Moreover, loss of LPP $\alpha 5$ exhibited a similar phenotype with pale yellow-green leaves. These results suggest that TOR-Raptor2-S6K1 is important for modulating thylakoid membrane lipid biosynthesis, homeostasis, thus enhancing thylakoid grana architecture and normal photosynthesis ability in rice.

Key words: Ribosomal protein S6 kinase (S6K), Target of rapamycin (TOR), Regulatory associated protein of TOR (Raptor), Galactolipid biosynthesis, Oryza sativa

\section{Introduction}

Target of rapamycin (TOR) is a Ser/Thr protein kinase belonging to the phosphoinositide 3-kinase-related kinase family, and its activity is inhibited by rapamycin, an immunosuppressant drug [1]. TOR signaling is a central regulator of growth and development through the modulation of protein synthesis, ribosome biogenesis, metabolism, and autophagy in response to growth factors, nutrient availability, energy status, and stress in yeast and animals [1-3]. Two TOR complexes, TORC1 and TORC2, have been identified in both yeast and animals. TORC1, which consists of TOR, Regulatory associated protein of TOR (Raptor), and LST8, is highly conserved in yeast, animals and plants. TORC1 is sensitive to rapamycin and senses different signals such as growth factors, amino acids, energy status, and oxygen to modulate cell growth and various metabolism processes in diverse eukaryotes [1,2,4-7]. The loss of core components such as TOR and Raptor in TORC1 results in embryonic lethality in worms, flies, mice, and Arabidopsis [8-13]. By comparison, TORC2 consists of mTOR, rapamycin-insensitive companion of mTOR (Rictor), and mLST8. TORC2 is insensitive to rapamycin and plays a major role in regulating cell survival and cytoskeletal organization in response to growth factors $[1,2]$.

The ribosomal protein S6 kinase (S6K), a Ser/Thr AGC kinase, is a downstream component of TORC1 signaling involved in cell growth, cell proliferation, and stress response via modulating protein synthesis and ribosomal biogenesis. S6K phosphorylates the S6 protein of the 40S ribosomal subunit to enhance mRNA translation via the recruitment of tRNAs to mRNA as well as translation initiation factors, thus promoting cell proliferation and cell expansion in animal cells [1,14]. The loss of S6K1 in mice and Drosophila resulted in small cell size and body [15,16], and $s 6 k 1 / s 6 k 2$ double mutants 
exhibited perinatal lethality in mice [17]. S6K1 deletion resulted in reduced muscle cytoplasmic volume via control of the muscle cell cycle [18]. The evidence suggests that S6K is essential for growth and development in animals. Arabidopsis possesses two S6K genes, AtS6K1 and AtS6K2, which are located in tandem on chromosome 3. AtS6K1 activity can be inhibited by osmotic stress in a TOR/Raptor-dependent manner, and AtS6K1 overexpression confers greater sensitivity to osmotic stress in germinating seeds [19]. S6K plays an important role in chromosome stability and functions as a repressor of cell proliferation in Arabidopsis. Partial loss of S6K results in various sizes of organs, with increased ploidy and aneuploidy as well as reduced pollen viability [20]. Arabidopsis S6K1 activation by TOR is important for reinitiating the translation of mRNA that contains upstream open reading frames in the $5^{\prime}$-untranslated regions, and this process is enhanced by auxin [21,22]. S6K from maize is activated by insulin or insulin-like growth factors (IGFs) through TOR signaling to promote cell growth [23-25]. These results suggest that the plant S6K is important for cell proliferation and the osmotic stress response.

Lipids function not only as high-condensate energy storage components, but also as building blocks for membrane structure and signaling molecules in cells. Photosynthesis occurs in the thylakoid grana of chloroplasts; the stacks of membrane lipids provide a fundamental and critical backbone structure for thylakoid grana architecture to maintain normal photosynthesis. Galactolipids, such as monogalactosyldiacylglycerol (MGDG) and digalactosyldiacylglycerol (DGDG), are the most abundant building block lipids in thylakoid membranes. They constitute $\sim 52 \%$ and $\sim 27 \%$ of chloroplast glycerolipids in leaf tissue, respectively, and are indispensable for photoautotrophic growth [26]. The loss of critical enzymes catalyzing the synthesis of MGDG and DGDG results in impaired photosynthesis, thus conferring plant lethality during an early stage in Arabidopsis [27-29].

Despite its biological significance, knowledge regarding the upstream signaling in regulating galactolipid synthesis is very limited. TOR signaling modulates cell growth through the activation of various anabolic processes [30]. Alterations of core components, such as TOR and S6K1, resulted in substantial changes in protein synthesis and lipogenesis in animals [1,31-33]. Increasing evidence has shown that TOR-S6K1 plays an important role in lipid anabolic process in animal cells. Genetic or pharmacological suppressed core components in TOR-S6K1 pathway led to reduced lipogenesis and adiposity in mammalian 
cells. S6K1 knockout mutant exhibited lean phenotype under normal and high-fat diets in mice [34, 35]. Suppression of Raptor by RNA interference also inhibited adipogenesis in preadipocytes [36]. Raptor knockout mice decreased the accumulation of triacylglycerol (TAG) and cholesterol in liver on a Western diet [37]. Rapamycin treatment or suppressed S6K1 by shRNA in embryoid bodies hampers the formation of preadipocytes in mice [34]. Depleted dTOR signaling in the fat body conferred a significant larval growth defect in Drosophila [38]. The role of TOR-Raptor-S6K1 in lipid metabolism remains elusive in plants, particularly crop plants. A recent study on global metabolite profiling showed that the content and fatty acid species of TAG were altered when TOR activity was suppressed by rapamycin in Arabidopsis [39], suggesting its role in lipid metabolism in plants. However, our understanding toward the TOR signaling with specific component modulating a specific lipid metabolism pathway remains limited in plants.

Here, we provide genetic and pharmacological evidence that TOR-Raptor2-S6K1 is important in regulating galactolipid MGDG and DGDG synthesis to modulate thylakoid grana architecture for photosynthesis. Genetic suppression of $S 6 K 1$ in rice resulted in pale yellow-green leaves, defective thylakoid grana architecture. Enzymatic assay showed that rice $S 6 K 1$ encodes a functional protein kinase, which is closely related to human p70S6K. S6K1 interacts with Raptor2 and is regulated by Raptor2 and TOR activity. Genetically disrupting Raptor2 or pharmacologically suppressing TOR activity by the inhibitors mimicked the S6K1-depleted phenotype. Lipid profiling revealed that TOR-Raptor2-S6K1 was important for galactolipid biosynthesis and fatty acid homeostasis through the transcriptional modulation to facilitate thylakoid grana modeling. Transcriptional analysis from $s 6 k 1$, raptor 2 mutants or TOR suppressed plants showed a significant reduced expression levels of one set of genes, such as lipid phosphate phosphatase $\alpha 5$ (LPP $\alpha 5$ ), $M G D G$ synthase 1 (MGDI), and DGDG synthase 1 (DGD1), involved in galactolipid synthesis, and a loss of LPP $\alpha 5$ exhibited a similar phenotype with pale green leaves.

\section{Materials and Methods}

\subsection{Plant materials and growth conditions}


All plants including the T-DNA insertion mutants, RNAi silencing, and overexpressed lines used in this study were derived from Japonica (Oryza sativa) cv Dongjin. Plants were grown in soil at the experimental station or in the greenhouse at Huazhong Agricultural University (Wuhan, China). Seedlings were grown in medium with or without TOR inhibitors Torin 2 or AZD8055 in growth room under $14 \mathrm{~h}$ light $\left(30^{\circ} \mathrm{C}\right) / 10$ $\mathrm{h}$ dark $\left(26^{\circ} \mathrm{C}\right)$, photosynthetic photon flux density of $450-600 \mu \mathrm{mol} \cdot \mathrm{m}^{-2} \cdot \mathrm{s}^{-1}$, and $50 \%$ relative humidity.

\subsection{Mutant isolation and complementation test}

The seeds of mutants containing the T-DNA insertion were obtained from the POSTECH RISD (http://www.postech.ac.kr/life/pfg/risd/). Homozygous mutants were isolated using the T-DNA left border primer coupled with gene-specific primers. For $s 6 \mathrm{kl}$ mutant complementation, the $5.6 \mathrm{~kb}$ genomic DNA, including the full-length $S 6 K 1$ gene $(4.1 \mathrm{~kb})$ and its native promoter region $(1.5 \mathrm{~kb})$, was amplified from rice leaf DNA and then cloned into the pCAMBIA 2301 binary vector digested with SacI and SalI. The constructs were then introduced into homozygous mutants by Agrobacterium-mediated transformation. The rice calli derived from mature embryos were used for transformation to induce transgenic regeneration plants [40]. The complementation plants were confirmed by PCR using the pCAMBIA 2301 border primer coupled with gene-specific primers. All primers used in this study for gene cloning, vector construction, and genotyping are listed in Supplementary Table S1.

\subsection{RNA extraction, RT-PCR, and quantitative real-time PCR}

Total RNA was extracted from various tissues using Trizol reagent (TransGene Biotech, Beijing, China). DNA was removed by treatment with RNase free DNaseI. The first strand cDNAs were synthesized from RNA templates using TransScript cDNA Synthesis SuperMix kit (TransGene Biotech). The cDNA concentration was adjusted to a similar level based on the $\mathrm{Ct}$ value for $\beta$-actin (LOC_Os01g16414) by quantitative real-time PCR. The resultant cDNAs were used for cloning or gene expression profiling. Quantitative real-time PCR was monitored with a MyIQ real time PCR system (Bio-Rad) via using Top Green qPCR SuperMix (TransGene Biotech). Rice $\beta$-actin was used as the 
internal standard to evaluate the relative levels of gene expressions. Quantitative real-time PCR was run under the following conditions: $95^{\circ} \mathrm{C}$ for $1 \mathrm{~min} ; 40$ cycles of $95^{\circ} \mathrm{C}$ for $30 \mathrm{~s}$, $55^{\circ} \mathrm{C}$ for $30 \mathrm{~s}, 72^{\circ} \mathrm{C}$ for $30 \mathrm{~s}$; and $72^{\circ} \mathrm{C}$ for $10 \mathrm{~min}$ for final extension of DNA. All primers for quantitative real-time PCR are listed in Supplementary Table S2.

\subsection{Measurements of photosynthesis and starch content}

The photosynthesis rate was measured from plants grown in the field at the tillering stage by a portable photosynthesis device (Yaxin-1102, Beijing). From 9:00 a.m. to 11:30 a.m. on sunny days the fully expanded and actively growing leaves with similar age and position were enclosed in the seal chamber of the photosynthesis device under sunlight for 5 minutes. The photosynthesis rate was based on a reduced $\mathrm{CO}_{2}$ concentration in the chamber per unit of time and was calculated automatically by the device.

For starch measurement, the leaf sample was homogenized and extracted with $80 \%$ ethanol at $80^{\circ} \mathrm{C}$ for 30 minutes to remove soluble sugars. The remaining sediment was gelatinized in $100^{\circ} \mathrm{C}$ for 15 minutes and then incubated with perchloric acid to extract starch for three times. The extract ( $50 \mu \mathrm{l})$ was mixed with $450 \mu \mathrm{l}$ of sulfuric acid containing anthrone $(2 \mathrm{mg} / \mathrm{ml})$ at $95^{\circ} \mathrm{C}$; the absorbance at $625 \mathrm{~nm}$ was then monitored by spectrometer (Infinite M200 PRD, Switzerland).

\subsection{Subcellular localization}

The full length cDNAs of rice S6K1 and S6K2 fused with GFP tag at the 5'- end were cloned into pCAMBIA1301S vector digested by KpnI and BamHI. Agrobacterium GV3101 containing the constructs was infiltrated into tobacco leaves for transient expressions of GFP-S6Ks. The GFP-S6K fusion proteins were monitored under a confocal laser scanning microscopy (Leica TCSSP2).

\subsection{Lipid separation by thin layer chromatography (TLC) and quantitative profiling}

Lipids were extracted from leaves of rice plants at the seedling and tillering stages, respectively. Leaf samples were quickly immersed in $75^{\circ} \mathrm{C}$ isopropanol with $0.01 \%$ 
butylated hydroxytoluene (BHT) for 15 minutes, followed by the addition of chloroform: water $(2.5: 1, \mathrm{v} / \mathrm{v})$ to extract lipids for $1 \mathrm{~h}$ at room temperature by shaking. The extracts were transferred to a clean tube, and remaining leaves were further extracted with chloroform : methanol $(2: 1, \mathrm{v} / \mathrm{v})$ containing $0.01 \%$ BHT at room temperature for several times until the leaves became completely bleached. The extracts were combined and washed with $1 \mathrm{M} \mathrm{KCl}$ and water successively, followed by evaporation with a stream of nitrogen. The resultant lipids were separated on TLC using the developing solvent composed of acetone, toluene, and water (91:30:7.5, v/v) [41]. The spots in the TLC plate were scraped out and methylated in methanol containing $1 \% \mathrm{H}_{2} \mathrm{SO}_{4}$ and $0.05 \%$ BHT at $80^{\circ} \mathrm{C}$ for 30 minutes, and then quantitatively analyzed by GC-MS or GC measurement using glyceryl triheptadecanoate (Sigma-Aldrich) as the internal standard. The GC-MS running conditions were as follows: The temperature for injection port was $250^{\circ} \mathrm{C}$ with the pressure of $11.46 \mathrm{psi}$ and the split ratio of $10: 1$; the oven temperature initiated at $140^{\circ} \mathrm{C}$ for $2 \mathrm{~min}$ and was then increased by $10^{\circ} \mathrm{C} / \mathrm{min}$ up to $180^{\circ} \mathrm{C}$ for $3 \mathrm{~min} ; 4^{\circ} \mathrm{C} / \mathrm{min}$ up to $230^{\circ} \mathrm{C}$ for $2 \mathrm{~min}$; and finally by $20^{\circ} \mathrm{C} / \mathrm{min}$ up to $300^{\circ} \mathrm{C}$ for $5 \mathrm{~min}$. The total flow rate was $13 \mathrm{ml} / \mathrm{min}$ and the column flow rate was $0.19 \mathrm{ml} / \mathrm{min}$. The $\mathrm{GC}$ was run under the following conditions: The temperature for injection port was set at $180^{\circ} \mathrm{C}$ with the pressure at 16.06 psi and the split ratio of 20:1. The oven temperature was initiated at $180^{\circ} \mathrm{C}$ for $2 \mathrm{~min}$ and then was increased by $10^{\circ} \mathrm{C} / \mathrm{min}$ up to $220^{\circ} \mathrm{C}$ for $5 \mathrm{~min}$. The temperature of the flame ionization detector is $280^{\circ} \mathrm{C}$ with the flow rate of 30,300 , and $25 \mathrm{ml} / \mathrm{min}$ for hydrogen, air, and helium, respectively.

\subsection{Protein expression, purification, S6K1 and TOR kinase activity assays}

The full-length cDNA of $S 6 K 1$ was amplified from rice leaf tissue and ligated into KpnI- and BamHI-digested sites of the pCAMBIA1301U vector under the control of the maize ubiquitin promoter. The construct was transformed into Japonica cv Dongjin by Agrobacterium-mediated transformation. Proteins were extracted from rice transgenic calli containing S6K1-FLAG using buffer B (50 mM Tris-HCl, pH 7.4, $150 \mathrm{mM} \mathrm{NaCl,} 1 \mathrm{mM}$ PMSF, $0.1 \%$ Triton X-100, $2 \mathrm{mg} / \mathrm{ml}$ Protease Inhibitor, $50 \mathrm{mM}$ sucrose), followed by centrifugation at $10,000 \mathrm{~g}$ for $20 \mathrm{~min}$. The supernatant was incubated with anti-FLAG M2 affinity gel (Sigma-Aldrich) for 3 hours at $4^{\circ} \mathrm{C}$, and then the beads were washed with TBS (50 mM Tris-HCl, pH 7.4, $150 \mathrm{mM} \mathrm{NaCl)} \mathrm{3-5} \mathrm{times} \mathrm{to} \mathrm{obtain} \mathrm{purified} \mathrm{S6K1-FLAG.} \mathrm{The}$ 
protein was eluted by incubation with elution buffer $(100 \mathrm{mM}$ glycine $\mathrm{HCl}, \mathrm{pH} 3.5)$ for 5 min at room temperature. After a short centrifugation, the supernatant was immediately transferred to a clean tube containing $10 \times$ buffer $(0.5 \mathrm{M}$ Tris- $\mathrm{HCl}, \mathrm{pH} 7.4,1.5 \mathrm{M} \mathrm{NaCl})$ at $4^{\circ} \mathrm{C}$ for further assay or storage. S6K1 kinase activity was analyzed with an S6K activity assay kit (Enzo Life Sciences) according to the manufacturer's instructions. Briefly, the purified S6K1 protein was added to reaction buffer $(50 \mathrm{mM}$ Tris-HCl, $\mathrm{pH} 7.5,10 \mathrm{mM}$ $\mathrm{MgCl}_{2}, 1 \mathrm{mM}$ DTT, $0.2 \mathrm{mM} \mathrm{ATP}$ ) and incubated in a 96-well plate coated with the S6K substrate, a synthesized peptide containing the S6 consensus peptide (KRRRLASLR), for various time intervals. After removal of the reaction mixture and three washes with PBST buffer $\left(137 \mathrm{mM} \mathrm{NaCl}, 2.7 \mathrm{mM} \mathrm{KCl}, 10 \mathrm{mM} \mathrm{Na} 2 \mathrm{HPO}_{4}, 2 \mathrm{mM} \mathrm{KH}_{2} \mathrm{PO}_{4}, \mathrm{pH}\right.$ 7.4, 0.05\% Tween 20, v/v), the plate was incubated with anti-phospho-S6 antibody (1:1000) for $1 \mathrm{~h}$, followed by a secondary antibody conjugated to horseradish peroxidase (HRP) for $20 \mathrm{~min}$. S6K1 kinase activity was monitored after color development by adding tetramethylbenzidine (TMB) substrate with a microplate reader (TECAN Infinite M200 Pro) and measuring the absorbance at $450 \mathrm{~nm}$. Proteins purified from WT or plants expressing the pCAMBIA1301U vector were used as negative controls. The protein extracted from WT or plants containing vector only was subjected to the same procedure and used as a negative control.

For the TOR kinase activity assay, total proteins were extracted from the leaves of variously treated seedlings using buffer B. After centrifugation at 10,000 $\mathrm{g}$ for $20 \mathrm{~min}$ at $4^{\circ} \mathrm{C}$, the supernatant proteins were separated by $10 \%$ SDS-PAGE and then transferred to a polyvinylidene difluoride (PVDF) membrane. The membrane was blotted with anti-phospho-p70S6K (pThr389) antibodies (1:1000) (Cell Signaling Technology), followed by a secondary antibody conjugated with HRP (1:2000). The phosphorylation sites of rice S6K1, Arabidopsis S6K1, and human p70S6K1 are highly conserved. Rabbit polyclonal antibodies against human p70S6K (pThr389) have been shown to react with plant S6K proteins [42, 43]. After incubation with LumiGLO at room temperature, phosphorylated S6K (pThr389) was detected using a bioluminescent imaging instrument (LAS-4000).

\subsection{Immunoprecipitation}


To test the interaction between S6K1 and Raptor proteins in vivo, proteins were extracted from rice transgenic leaves containing the S6K1-FLAG fusion protein and immunoprecipitated using an anti-FLAG M2 affinity gel (Sigma-Aldrich). The proteins in the complex were separated by $10 \%$ SDS-PAGE and transferred to a PVDF membrane. The membrane was blotted with an anti-Human Raptor antibody (1:1000) (Proteintech), followed by a secondary antibody (1:2000) conjugated with alkaline phosphatase. Rice Raptor2 sequence is closely related to Human Raptor (Supplementary Fig. S1), and could be recognized by anti-Human Raptor antibody, which was verified by using rice Raptor2-His protein. The protein associated with S6K1 was visualized by an alkaline phosphatase reaction. To further confirm the interaction between the two proteins, the full-length cDNA of $S 6 K 1$ fused to the GST tag at the 5'-end was cloned into the EcoRI and XhoI sites of the pET42a vector (Novagen), and the full length cDNA of Raptor2 was ligated to the pET28a vector digested with EcoRI and XhoI. The resultant constructs were transformed into E. coli (BL21) cells to express the S6K1-GST and Raptor2-His recombinant proteins, respectively. S6K1-GST was incubated with Raptor2-His for $6 \mathrm{~h}$ at $4^{\circ} \mathrm{C}$, and the proteins were precipitated using a glutathione agarose affinity gel (Sigma-Aldrich); Raptor2-His fusion protein was detected using anti-His antibody. Furthermore, S6K1-FLAG was incubated with Raptor2-His, and the proteins were precipitated using an anti-FLAG M2 affinity gel (Sigma-Aldrich) for an interaction assay following a similar procedure. The protein extracted from cells containing vector only was subjected to the same procedure and used as a negative control.

\section{Results}

\subsection{S6K1 is expressed in active tissues and encodes a functional S6 kinase}

The rice genome contains two S6K genes, S6K1 and S6K2. To investigate the expression patterns of $\mathrm{S} 6 \mathrm{~K}$ genes in rice, RNA was extracted from various stages and tissues for semi-quantitative RT-PCR. The transcript levels of both $S 6 K 1$ and $S 6 K 2$ were highly expressed in leaves and roots at the seedling stage. During the tillering stage, $S 6 K 1$ remained highly expressed in both root and leaf tissues when compared with S6K2 (Fig. 1A). During the heading stage, the transcript level of $S 6 K 1$ in younger leaves was greater than that in mature leaves (Fig. 1A). Overall, $S 6 K 1$ expression level in the roots remained relatively consistent across various stages. By comparison, $S 6 K 1$ transcript level in leaves 
was higher than that of $S 6 K 2$ at the tillering and heading stages, and was highly expressed at the early stage, and then gradually decreased as plant development progressed (Fig. 1A). These data suggest that $S 6 K 1$ is highly expressed in actively growing tissues.

To determine the subcellular localization of rice S6Ks, full-length cDNAs encoding S6K1 and S6K2 were amplified from younger leaf tissues in rice. The green fluorescent protein (GFP) was fused to the $\mathrm{N}$ terminus of the S6K, and the GFP-S6K fusion protein was transiently expressed in tobacco epidermal cells. GFP-S6K1 was detected in both the cytoplasm and nucleus, whereas GFP-S6K2 was localized mainly in the nucleus (Fig. 1B). To further confirm the subcellular localization, a FLAG tag was fused to the C-terminus of S6K1 and then expressed in rice plants. The protein localization to various cell compartments was separated by two-phase partitioning and detected using a FLAG antibody. The result showed that S6K1-FLAG was detected in the soluble, plasma membrane and intracellular membrane fractions (Fig. 1C), which is consistent with results for GFP-S6K1 visualized under a confocal laser scanning microscopy. Furthermore, Rice S6K1 purified by affinity chromatography displayed a high activity toward a human ribosomal protein S6 consensus polypeptide (Fig 1D), suggesting that rice $S 6 K 1$ encodes an active S6 kinase that is closely related to mammalian p70S6K.

\subsection{Genetic suppression of S6K1 causes pale yellow-green leaves}

To elucidate the biological functions of rice S6K1 in growth and development, a mutant designated $s 6 k 1$, caused by a T-DNA insertion into $S 6 K 1$, was identified. The T-DNA is located in the promoter region 2427 bp upstream of the translation start site (Fig. 2A). The homozygous mutant $s 6 k 1$ was isolated by PCR screening using a T-DNA left border primer and $S 6 K 1$-specific primer (Fig. 2B). The $S 6 K 1$ transcript in $s 6 k 1$ remains detectable but is substantially reduced compared with that of wild type (WT) plants (Fig. 2C). The $s 6 k 1$ plants exhibited pale yellow-green leaves under normal growth conditions (Fig. 2D-F). The chlorophyll a content of the $s 6 k 1$ leaves was reduced by $29 \%$ compared with that of WT during the tillering stage (Fig. 2G). The contents of chlorophyll b and carotenoid in s6kl were also significantly lower than those in WT (Fig. 2G). The photosynthetic rate in $s 6 \mathrm{kl}$ plants was only $27 \%$ of that in WT plants grown in the field during sunny days (Fig. $2 \mathrm{H}$ ). The starch content in the leaves of $s 6 k 1$ was significantly 
reduced to $57 \%$ of WT, and thousand-grain weight in the s6k1 mutant was only 58\% of WT (Fig. 2I,J).

To confirm these phenotypic alterations caused by the depletion of S6K1, the S6K1 gene driven by its native promoter was introduced into the $s 6 k 1$ to obtain genetic complementary (COM) plants. The pale yellow-green leaves in $s 6 k 1$ compensated by $S 6 K 1$ (COM) plants were restored to a normal green color like WT, and the pigment contents were completely reversed (Fig. 2D-G). The s6kl mutant plants containing the $56 K 1$ gene controlled by the ubiquitin promoter (OE) also exhibited normal green leaves as shown in WT (Fig. 2E). These data demonstrate that the pale yellow-green leaves are caused by a S6K1 deficiency and that S6K1 is important for normal green leaf phenotype in rice.

\subsection{S6K1-depleted plants exhibit defect in thylakoid grana architecture}

The pale yellow-green leaf phenotype in the $s 6 \mathrm{k} 1$ mutant prompted us to investigate whether S6K1 was involved in chloroplast biogenesis. The leaf tissues of $s 6 k 1$ and WT plants were examined under transmission electron microscopy (TEM) during the seedling and tillering stages. The architecture of the thylakoid grana in the chloroplasts was defective in $s 6 k 1$. The thickness of the cylindrical grana stacks in $s 6 k 1$ was dramatically reduced; the shape of the thylakoid grana was barely observed in the chloroplasts of $s 6 k l$, whereas the intact, tightly organized and much more lamellaes of thylakoid grana were detected in the chloroplasts of WT leaves at the seedling and the tillering stages (Fig. 3A, B). Instead of intact cylindrical grana stacks, 18 plastoglobules on average were detected within each chloroplast in $s 6 \mathrm{Kl}$; whereas only $\sim 3$ droplets per chloroplast were found in WT (Fig. 3A, B, D). The defect in the grana stacking structure and the increased amount of plastoglobules in $s 6 k 1$ indicate that S6K1 plays an important role in thylakoid membrane biogenesis and maintenance, thus enhancing photosynthesis in rice. Indeed, the loss of well-organized thylakoid grana in $s 6 \mathrm{kl}$ plants resulted in reduced starch granules within the chloroplasts by ultrastructural observation. WT chloroplasts contained a large amount of starch granules, whereas starch granules were almost undetectable in the chloroplasts of $s 6 k 1$ at the tillering stage (Fig. 3B). This observation is consistent with the result of the starch content (Fig. 2I). In addition, the chloroplast size in the $s 6 \mathrm{kl}$ mutant was also smaller than that of WT (Fig. 3C). These data suggest that S6K1 plays a positive role in thylakoid 
membrane modeling and enhances photosynthesis for carbohydrate accumulation in rice.

\subsection{S6K1 is important for thylakoid membrane biosynthesis and fatty acid homeostasis}

The defects in thylakoid grana architecture and thylakoid membranes in $s 6 k 1$ indicate that S6K1 may modulate lipid metabolism. The contents of galactolipids MGDG and DGDG were significantly reduced in $s 6 k 1$ mutant, whereas phospholipids such as phosphatidylcholine (PC), phosphatidylethanolamine (PE), and phosphatidylglycerol (PG) remained unchanged in $s 6 k 1$ plants, as compared to WT at the seedling stage (Fig. 4A). The contents (nmol/mg.dry weight) of MGDG and DGDG in $s 6 \mathrm{kl}$ were reduced by $42 \%$ and 44\%, respectively (Fig. 4A). Rice MGDG and DGDG were composed primarily of 18:3, 18:1, 18:0, and 16:0 fatty acids at the seedling stage (Fig. 4B, C). The reduced MGDG and DGDG in $s 6 k 1$ plants resulted from decreased levels of 16:0, 18:0, and 18:1 fatty acid species. The absolute content of polyunsaturated fatty acid 18:3 of MGDG and DGDG in $s 6 k 1$ mutant was also reduced compared with WT, but the decreased amount was smaller than that of 16:0, 18:0, and 18:1 fatty acids (Fig. 4B, C). Thus, the relative amount of polyunsaturated fatty acid (18:3) of galactolipids was increased in the $s 6 k 1$ mutant; the ratio of $18: 3$ to total fatty acids of MGDG was $78 \%$ in the $s 6 k 1$ mutant in contrast to $59.6 \%$ in WT. The defective galactolipids and altered fatty acid composition were rescued by the S6K1 complementation (Fig. 4A-C), which further support the fact that S6K1 is important for thylakoid membrane biosynthesis and appropriate fatty acid composition.

\subsection{S6K1 directly interacts with Raptor2 to modulate thylakoid membrane biosynthesis}

Raptor is a core component in TORC1 functions as upstream of S6K1. To explore the upstream regulator of S6K1, FLAG-tagged S6K1 was steadily expressed in rice plants, and was co-immunoprecipitated by using anti-FLAG antibody covalently attached to agarose. The endogenous Raptor protein was found to be associated with S6K1, as detected by Raptor antibodies, whereas negative control containing FLAG failed to bind to the Raptor protein (Fig. 5A). To further confirm the interaction, S6K1-GST (glutathione S-transferase) and Raptor2-His were expressed in E. coli cells, and the protein complex was pulled down using glutathione covalently conjugated to agarose beads. The proteins in the complex were separated by SDS-PAGE, and the resultant protein was detected using a His 
antibody. Raptor2 was associated with S6K1, but not with the GST control, which demonstrated that S6K1 interacts directly with Raptor2 (Fig. 5B). Moreover, an S6K1-FLAG fusion protein expressed in rice plants also interacted with Raptor2-His (Fig. $5 \mathrm{C})$.

The interaction between S6K1 and Raptor2 indicates that Raptor2 is likely involved in thylakoid membrane biosynthesis. To explore the cellular functions of Raptor2, a deficiency mutant raptor 2 was isolated from the rice. The raptor 2 mutant is caused by a T-DNA insertion in the third intron of Raptor2 (Fig. 6A), and the Raptor 2 mRNA in raptor 2 is undetectable by semi-quantitative RT-PCR (Fig. 6B), indicating that it is a null mutant. The raptor 2 mutant exhibited pale or striped-pale leaves with substantially reduced chlorophyll content at the seedling and tillering stages (Fig. 6C, D). The contents of chlorophyll a, b, and carotenoid in striped-pale leaves of raptor 2 were $55 \%, 58 \%$, and $58 \%$ of those in WT, respectively, at the tillering stage (Fig. 6G). The pale leaves exhibited defective thylakoid grana structure in the raptor2 mutant (Fig. $6 \mathrm{H}-\mathrm{J}$ ). The phenotype of the raptor 2 mutant was more severe than that of the $s 6 k 1$ mutant, and raptor 2 plants exhibited complete loss of chloroplasts in some cells at the tillering stage (Fig. $6 \mathrm{~J}$ ). The results indicate that Raptor2 is also involved in thylakoid membrane biosynthesis and maintenance. Moreover, plants with substantially suppressed Raptor 2 mRNA by RNAi silencing also exhibited a similar phenotype with pale leaves (Fig. 6E, F). The galactolipids MGDG and DGDG were significantly reduced in leaves of the raptor 2 mutant; the contents of MGDG and DGDG in raptor2 were decreased by $60 \%$ and $46 \%$, respectively, compared with WT at the seedling stage (Fig. 6N). The S6K1 transcript level was not down-regulated in the raptor2 mutant (Fig. 6L). However, loss of Raptor2 resulted in the reduction of S6K phosphorylation level and S6K activity, as compared with WT (Fig. 6K, M), suggesting that Raptor2 interacts with S6K1 to stimulate the S6K phosphorylation and activity. The common feature with defective thylakoid grana architecture, accompanied by similar trend on alterations of galactolipid content, was found in both raptor 2 and $s 6 k 1$ mutants. This finding reveals that S6K1 interacts with Raptor2 to modulate coordinately thylakoid membrane biosynthesis and thylakoid grana modeling.

\subsection{Raptor2-S6K1 involved in galactolipid biosynthesis is regulated by TOR activity}

It was shown that Raptor is a core component of TOR signaling in animals and 
Arabidopsis [4,19]. To elucidate whether the involvement of Raptor2/S6K1 in thylakoid membrane biogenesis is regulated by TOR signaling, three-leaf-old seedlings were transferred to liquid media containing various concentrations of AZD8055 and Torin2, the newly developed ATP-competitive TOR-specific inhibitors $[44,45]$. The application of either AZD8055 or Torin2 resulted in pale yellow-green leaves, and the chlorophyll content was significantly reduced when compared to the seedlings grown under control condition without TOR inhibitor (Fig. 7A, B, D). The phosphorylated S6K was dramatically suppressed by TOR activity inhibitors with more prominent of AZD8055 treatment (Fig. 7C). By comparison, the total S6K1 level was relatively constant under these conditions tested (Fig. 7C). These results suggest that the effect of S6K1 on galactolipid synthesis is regulated by TOR-Raptor2.

To further test whether the suppression of TOR activity lead to the alteration of lipid content, lipids were extracted from leaves of seedlings grown in liquid medium containing AZD8055 or Torin2 $(30 \mu \mathrm{M})$ for 5 days. The seedlings grown in the same medium without TOR inhibitors were used as a control. The significant reductions of galactolipids MGDG and DGDG were found in AZD8055 or Torin2 treated seedlings. The contents of MGDG and DGDG in AZD8055 treated leaves were decreased by $40 \%$ and $46 \%$, respectively, compared with plants grown in the control condition without TOR inhibitor (Fig. 7E). The effect of Torin2 on lipid alteration exhibited a similar response (Fig. 7E). By comparison, the contents of phospholipids remained less changed, suggesting that TOR is specifically involved in galactolipid biogenesis rather than general effect on lipid metabolism.

\subsection{Suppressed S6K1, Raptor2, and TOR simultaneously down-regulated the set of genes} involved in galactolipid synthesis

Galactolipids such as MGDG and DGDG are major components of thylakoid menbranes. MGDG is synthesized from diacylglycerol (DAG) by MGDG synthase (MGD) and subsequently DGDG by DGD, while DAG is derived from phosphatidic acid (PA) by lipid phosphate phosphatase (LPP) or phospholipids by non-specific phospholipase C (NPC). Rice contains seven LPPas (LPPal-7), three MGDs (MGD1-3), five DGDs (DGD1-5), and four NPCs (NPC1-4). In animal cells, TOR-S6K1 plays a positive role in lipogenesis through transcriptional regulation. To explore how TOR-Raptor2-S6K1 
regulates galactolipid metabolism, the expression levels of genes involved in galactolipid biosynthesis were profiled from the $s 6 k 1$, raptor 2 mutants and TOR suppressed plants using WT plants without TOR inhibitor treatment as control (Fig. 8A-C). The expression levels of $M G D 1$ in $M G D$ family, $D G D 1$ in $D G D$ family, $L P P \alpha 5$ in $L P P \alpha$ family, and NPC2 were most abundant in WT plants without TOR inhibitor treatment, whereas the transcript levels of $M G D 2, D G D 2, L P P \alpha 1, L P P \alpha 3$, and $L P P \alpha 7$ were relatively low (Fig. 8A-C). Interestingly, the expression levels of $L P P \alpha 5, M G D 1$, and $D G D 1$ were all dramatically downregulated in both mutants of $s 6 k 1$ and raptor2, and plants with TOR inhibitor treatments as well, compared to the control WT plants (Fig. 8A-C). The expression levels of $M G D 2, D G D 2$, and $L P P \alpha 3$ were relatively low and remained unchanged in all conditions tested. The alteration of expression levels of genes such as MGD3, LPP $\alpha 1$, $L P P \alpha 2, L P P \alpha 4, L P P \alpha 6, L P P \alpha 7, N P C 1, N P C 2$, and $P L D \alpha 1$ exhibited inconsistent patterns in the $s 6 k 1$, raptor 2 and TOR inhibitor treated plants (Fig. 8A-C). To further confirm that the regulation of $L P P \alpha 5, M G D 1$, and $D G D 1$ is specifically down regulated by TOR-Raptor2-S6K1 suppression, rather than by a consequence of the general effect of pale leaves, the expression pattern of the set of genes was also profiled in a pale mutant $c d s 5$ caused by loss of cytidinediphosphate diacylglycerol synthase 5 (CDS5) involved in phosphatidylglycerol synthesis [46]. The expression levels of LPP $25, M G D 1$ and DGD1 were not significantly different between the $c d s 5$ mutant and WT plants (Fig. 8D). The results suggest that $L P P \alpha 5, M G D 1$ and $D G D 1$ were corporately regulated by TOR, Raptor2, and S6K1. MGD1 and DGD1 have been shown to play an important role in galactolipid synthesis in Arabidopsis [27-29]. To investigate whether LPP $\alpha 5$ is involved in this process, a mutant lppa5 was isolated and loss of LPP $\alpha 5$ led to pale yellow-green leaves with substantially reduced chlorophyll content (Fig. 8E-G). Based on the results from the current study, a work model for TOR-Raptor2-S6K1 in galactolipid synthesis and lipid homeostasis was proposed in Fig. 9. TOR, Raptor2, and S6K1 coordinately modulate the transcript levels of genes encoding LPP $\alpha 5$, MGD1, and DGD1 involved in thylakoid membrane biogenesis and lipid homeostasis in rice.

\section{Discussion}

\subsection{Rice S6K1 is functionally unique and is regulated by TOR-Raptor2}


S6K1 functions as a key point in the TOR pathway to regulate cell growth and metabolism in animals [1,2]. By comparison, knowledge about S6K is relatively limited in plants. Arabidopsis possesses two $S 6 K s$ and the single mutant does not exhibit visible phenotype; however, the homozygous double mutants $s 6 k 1 s 6 k 2$ cannot be recovered [20], which suggests that S6K1 and S6K2 may be functionally redundant in growth regulation and that $\mathrm{S} 6 \mathrm{~K}$ is essential for gametophyte or embryonic development. The rice genome also contains two S6K genes, $S 6 K 1$ and $S 6 K 2$. S6K1 was detected in all tissues but was most abundant in actively growing tissues and localized in both the cytoplasm and nucleus, which is distinct from nucleus localized S6K2. The subcellular localizations of S6K1 and S6K2 are similar to that of Arabidopsis S6Ks [19]. The enzymatic analysis showed that it is highly conserved between rice S6K1 and animal p70S6K. However, rice S6K1 appears to be unique, as single gene mutation in $S 6 K 1$ resulted in pale yellow-green leaf phenotype with defective thylakoid grana architecture, and decreased biomass. It was showed that hemizygous s6k1s6k2/++ mutant and S6K1 RNAi lines exhibited increased non-viable pollen and aborted seeds in Arabidopsis [20], which was also found in rice s6k1 mutant, suggesting some conservation and yet distinct role of S6K between two species. The results suggest that rice S6K1 is important for thylakoid grana modeling during postembryonic stages, and the loss of S6K1 is not compensated by S6K2 in rice. It would be interesting to determine the role of $\mathrm{S} 6 \mathrm{~K} 2$ in the future study.

Raptor is a core component of the TORC1 complex, acting as a scaffold to recruit the substrate of TOR kinase for phosphorylation [4,5]. In Arabidopsis, the loss of Raptor1 resulted in embryonic lethality, whereas disruption of Raptor2 did not exhibit a visible phenotype $[13,47]$. The interaction between S6K and Raptor was found in Arabidopsis and animal cells $[4,19]$. The current study demonstrated that rice S6K1 also directly interacted with Raptor2, and genetic suppression of Raptor2 significantly diminished S6K phosphorylation and S6K activity. Loss of Raptor2 mimicked the phenotype of the $s 6 \mathrm{kl}$ mutant and TOR inhibitor treatment. Rice contains two Raptors, and Raptor1 and Raptor2 share $99 \%$ of sequence identity, with the exception of the lack of 142 amino acids in $\mathrm{N}$ terminus of Raptor2 (Supplementary Fig. S1). A more severe phenotype with white leaves as well as a more severe defect thylakoid membrane in Raptor2 RNAi plants than in the raptor 2 knockout mutant plants indicates that Raptor 1 also may be involved in this process, as Raptor2 RNAi might also cause suppression of the Raptorl transcript level. The results indicate that the function of S6K1 in modulating thylakoid membrane synthesis is regulated 
by the TOR-Raptor pathway.

\subsection{TOR-Raptor2-S6K1 is important for thylakoid membrane biogenesis}

TOR modulates lipogenesis in transcript regulation manner mediated by lipid related transcript factor SREBP. It was showed that TOR promotes the processed and mature SREBP1 formation to facilitate its nuclear translocation to up-regulate the genes involved in fatty acid and sterol biosynthesis, and this process requires S6K1 and exhibits a rapamycin-dependent manner in animal cells [30]. Rapamycin suppressed TOR activity in Cyanidioschyzon merolae expressing yeast FKBP12 protein led to triacylglycerol accumulation by enhancing the transcript levels of genes encoding glycerol-3-phosphate acyltransferase and acyl-CoA:diacylglycerol acyltransferase (DGAT) [48]. The knowledge of TOR-Raptor-S6K in lipid metabolism is limited in plants. The current results show that suppression of any component of TOR-Raptor2-S6K1 leads to defective thylakoid grana architecture with a significant reduction in galactolipids accompanied by downregulation of genes specifically involved in MGDG and DGDG anabolism in rice. TOR-Raptor2-S6K1 may function as an upstream signaling to modulate galactolipid synthesis and fatty acid homeostasis, thus for thylakoid grana architecture modeling.

A recent study on global metabolite analysis revealed that TOR suppressed plants by estradiol inducible artificial microRNA exhibited increased polyunsaturated fatty acids of TAG in Arabidopsis [39]. In this study, the mutant s6kl plants exhibited reduced galactolipids with a higher ratio of polyunsaturated fatty acid 18:3 to total fatty acids in MGDG and DGDG. The data suggest that the galactolipid content and appropriate ratio of polyunsaturated fatty acid 18:3 regulated by TOR-Raptor2-S6K1 are important for thylakoid grana membrane modeling and architecture. Generally, a higher level of polyunsaturated fatty acids is beneficial for a lower temperature adaptation in plants $[49,50]$, whereas these fatty acids may have the opposite effect at a higher temperature. Rice plants prefer to grow under relatively higher temperature conditions. An increased ratio of 18:3 fatty acid of MGDG in $s 6 k 1$ plants may promote membrane fluidity and instability under the growth condition. Based on our observation, plants exhibited more severe pale yellow-green leaves when grown at a higher temperature than at a lower temperature in both the $s 6 k 1$ and raptor 2 mutants. Plastoglobules are plastid localized lipoprotein particles, and mainly contain prenyl-quinones and neutral lipids such as carotenoids, triacylglycerol 
and phytyl ester and lipid associated proteins including plastoglobulin [51]. The increased accumulation of plastoglobules was observed in etioplasts with poor thylakoid membrane biogenesis or in chloroplasts of the mutants with defective thylakoid formation [52,53]. Defective thylakoid membrane and more plastoglobules were found in chloroplasts of both $s 6 k 1$ and raptor 2 mutants, as compared to WT plants, suggesting that TOR-Raptor2-S6K1 signaling is important for thylakoid lipid anabolic process in rice. The results demonstrated that TOR-Raptor2-S6K1 is important for thylakoid membrane biogenesis, thus enhance photosynthesis in rice.

In animal cells, a recent study showed that the role of TOR in lipid metabolism is mediated by Lipin-1, an enzyme that catalyzes PA dephosphorylation to DAG [37]. The evidence for the involvement of TOR-Raptor2-S6K1 in thylakoid membrane lipid homeostasis is robust and significant in rice. The transcript levels of $L P P \alpha 5, M G D 1$, and DGD1 were all suppressed in raptor2, s6k1 mutants, and TOR inhibitor treated plants, indicating that these genes are specially regulated by TOR-Raptor2-S6K1. Genetic suppression of LPP $\alpha 5$ also led to a similar phenotype with pale yellow-green leaves. Arabidopsis MGD1 is essential for thylaloid membrane synthesis, whereas MGD2 and MGD3 are involved in lipid remodeling under phosphate starvation [28,54]. Arabidopsis MGD1 knockout mutant produced negligible amounts of both MGDG and DGDG, with a total loss of thylakoid membranes and defect in photosynthetic activities [28]. MGD1 mutation or suppression by DEX induction caused a significantly reduced MGDG content accompanied by defective thylakoid structure and undeveloped plastids in Arabidopsis [55,56]. Loss of MGD1 also led to pale yellow-green leaves and reduced MGDG level in rice [57]. DGD1 deficiency mutant displayed reduced DGDG level in Arabidopsis [27,58]. In agreement with these results, the mutants $s 6 k 1$, raptor 2 , and TOR inhibited plants exhibited substantial decrease in the transcript levels of genes MGD1, DGD2 and LPPa5 involved in galctolipid synthesis, implicating that TOR-Raptor2-S6K1 signaling functions as a upstream transcriptional regulator in galactolipid synthesis to modulate thylakoid membrane biogenesis. DAG produced by LPP $\alpha 5$ provides a precursor for galactolipid synthesis by MGD1 and DGD1 (Fig. 9). It would be interesting to characterize the function of LPP $\alpha 5$ involved in galactolipid synthesis in more detail in the future study.

\subsection{A feedback loop of TOR-Raptor2-S6K1 in lipid metabolism and lipid signaling}


598

599

600

601

602

603

604

605

606

607

608

609

610

611

612

613

614

615

616

617

618

619

620

621

622

623

624

625

626

627

628

629

630

Regulation of the TOR-Raptor2-S6K1 network is intricate, and current results indicate that TOR-Raptor2-S6K1 functions upstream to regulate membrane lipid anabolic process through transcriptional manner. Both the TOR and S6K1 were also activated by the lipid messenger PA in animal cells $[40,59,60]$. Our data showed that suppression of S6K1 caused a substantial increase in PLD $\alpha 1$ activity and its product, the PA level, in $s 6 k 1$ (Supplementary Fig. S2), which indicates that a reduced S6K1 level was compensated by elevated PA. PA derived from PLD $\alpha 3$ and PLD $\varepsilon$ activation promotes plant growth $[42,61]$. The elevated PA in the $s 6 k 1$ mutant implicates that higher PA in the $s 6 k 1$ mutant may compensate for the reduced S6K1 level and allow plant growth to a certain extent. TOR-Raptor2-S6K1 may function as a feedback loop between lipid metabolism and lipid signaling.

\subsection{The dual effects of TOR-Raptor2-S6K1 on sugar accumulation and sensing}

Plants display an autotrophic manner through photosynthesis to produce carbohydrates that provide the basic backbone for the synthesis of various organic derivations for growth and development. The defect in thylakoid membrane biogenesis and photosynthesis in the S6K1-suppressed mutant resulted in the reduction of sugar accumulation and seed weight. In addition, the suppressed TOR activity by the inhibitors could be partially reversed by supplying sugar in the growth medium (Fig. 7C). TOR signaling is essential for sugar sensing to regulate root growth in Arabidopsis [43]. Glucose produced from photosynthesis is required for the activation of TOR signaling [43]. TOR-Raptor2-S6K1 appears to have double effects including sugar accumulation and sugar sensing.

\section{Supplementary information}

Supplementary Fig S1. Sequence alignment of Raptor proteins from Arabidopsis, rice and human.

Supplementary Fig. S2. The effect of S6K1 on PLD $\alpha$ activity and PA formation.

Supplementary Table S1. Primers for genotyping and vector constructions

Supplementary Table S2. Primers used for RT-PCR and quantitative real-time PCR 
631

632

633

634

635

636

637

638

639

640

641

642

643

644

645

646

647

648

649

650

651

652

653

654

655

656

657

658

659

660

661

662

663

\section{Conflict of interests}

The authors have no conflicts of interests.

\section{Acknowledgments}

This work was supported by grants from the Chinese National Key Basic Research Project (2012CB114200) and the National Science Foundation of China (31271514).

\section{References}

[1] S. Wullschleger, R. Loewith, M.N. Hall, TOR signaling in growth and metabolism, Cell 124 (2006) 471-484.

[2] M. Laplante, D.M. Sabatini, mTOR signaling in growth control and disease, Cell 149 (2012) 274-293.

[3] K. Maegawa, R. Takii, T. Ushimaru, A. Kozaki, Evolutionary conservation of TORC1 components, TOR, Raptor, and LST8, between rice and yeast, Mol. Genet. Genomics 26 (2015) 313-322.

[4] K. Hara, Y. Maruki, X. Long, K. Yoshino, N. Oshiro, S. Hidayat, C. Tokunaga, J. Avruch, K. Yonezawa, Raptor, a binding partner of target of rapamycin (TOR), mediates TOR action, Cell 110 (2002) 177-189.

[5] D.H. Kim, D.D. Sarbassov, S.M. Ali, J.E. King, R.R. Latek, H. Erdjument-Bromage, P. Tempst, D.M. Sabatini, mTOR interacts with raptor to form a nutrient-sensitive complex that signals to the cell growth machinery, Cell 110 (2002) 163-175.

[6] R. Loewith, E. Jacinto, S. Wullschleger, A. Lorberg, J.L. Crespo, D. Bonenfant, W. Oppliger, P. Jenoe, M.N. Hall, Two TOR complexes, only one of which is rapamycin sensitive, have distinct roles in cell growth control, Mol. Cell 10 (2002) 457-468.

[7] D.E. Martin, A. Soulard, M.N. Hall, TOR regulates ribosomal protein gene expression via PKA and the forkhead transcription factor FHL1, Cell 119 (2004) 969-979.

[8] H. Zhang, J.P. Stallock, J.C. Ng, C. Reinhard, T.P. Neufeld, Regulation of cellular growth by the Drosophila target of rapamycin dTOR, Gene. Dev. 14 (2000) 2712-2724.

[9] X. Long, C. Spycher, Z.S. Han, A.M. Rose, F. Müller, J. Avruch, TOR deficiency in C. elegans causes developmental arrest and intestinal atrophy by inhibition of mRNA translation, Cur. Biol. 12 (2002) 1448-1461.

[10] B. Menand, T. Desnos, L. Nussaume, F. Berger, D. Bouchez, C. Meyer, C. Robaglia, 
Expression and disruption of the Arabidopsis TOR (Target Of Rapamycin) gene, Proc. Natl. Acad. Sci. U. S. A. 99 (2002) 6422-6427.

[11] Y.G. Gangloff, M. Mueller, S.G. Dann, P. Svoboda, M. Sticker, J.F. Spetz, S.H. Um, E.J. Brown, S. Cereghini, G. Thomas, Disruption of the mouse mTOR gene leads to early postimplantation lethality and prohibits embryonic stem cell development, Mol. Cell. Biol. 24 (2004) 9508-9516.

[12] M. Murakami, T. Ichisaka, M. Maeda, N. Oshiro, K. Hara, F. Edenhofer, H. Kiyama, K. Yonezawa, S. Yamanaka, mTOR is essential for growth and proliferation in early mouse embryos and embryonic stem cells, Mol. Cell. Biol. 24 (2004) 6710-6718.

[13] D. Deprost, H.N. Truong, C. Robaglia, C. Meyer, An Arabidopsis homolog of RAPTOR/KOG1 is essential for early embryo development, Biochem. Bioph. Res. Co. 326 (2005) 844-850.

[14] D.C. Fingar, S. Salama, C. Tsou, E. Harlow, J. Blenis, Mammalian cell size is controlled by mTOR and its downstream targets S6K1 and 4EBP1/eIF4E, Gene. Dev. 16 (2002) 1472-1487.

[15] H. Shima, M. Pende, Y. Chen, S. Fumagalli, G. Thomas, S.C. Kozma, Disruption of the p70s6k/p85s6k gene reveals a small mouse phenotype and a new functional S6 kinase, EMBO J. 17 (1998) 6649-6659.

[16] J. Montagne, M.J. Stewart, H. Stocker, E. Hafen, S.C. Kozma, G. Thomas, Drosophila S6 kinase: a regulator of cell size, Science 285 (1999) 2126-2129.

[17] M. Pende, S.H. Um, V. Mieulet, M. Sticker, V.L. Goss, J. Mestan, M. Mueller, S. Fumagalli, S.C. Kozma, G. Thomas, S6 $\mathrm{K}^{-/-} / \mathrm{S} 6 \mathrm{~K} 2^{-/-}$mice exhibit perinatal lethality and rapamycin-sensitive 5'-terminal oligopyrimidine mRNA translation and reveals a mitogen-activated protein kinase-dependent S6 kinase pathway, Mol. Cell. Biol. 24 (2004) 3112-3124.

[18] M. Ohanna, A.K. Sobering, T. Lapointe, L. Lorenzo, C. Praud, E. Petroulakis, N. Sonenberg, P.A. Kelly, A. Sotiropoulos, M. Pende, Atrophy of S6K1-/- skeletal muscle cells reveals distinct mTOR effectors for cell cycle and size control, Nat. Cell Biol. 7 (2005) 286-294.

[19] M.M. Mahfouz, S. Kim, A.J. Delauney, D.P. Verma, Arabidopsis TARGET OF RAPAMYCIN interacts with RAPTOR, which regulates the activity of S6 kinase in response to osmotic stress signals, Plant Cell 18 (2006) 477-490.

[20] R. Henriques, Z. Magyar, A. Monardes, S. Khan, C. Zalejski, J. Orellana, L. Szabados, C. de la Torre, C. Koncz, L. Bögre, Arabidopsis S6 kinase mutants display 
chromosome instability and altered RBR1-E2F pathway activity, EMBO J. 29 (2010) 2979-2993.

[21] M. Schepetilnikov, K. Kobayashi, A. Geldreich, C. Caranta, C. Robaglia, M. Keller, L.A. Ryabova, Viral factor TAV recruits TOR/S6K1 signalling to activate reinitiation after long ORF translation, EMBO J. 30 (2011) 1343-1356.

[22] M. Schepetilnikov, M. Dimitrova, E. Mancera-Martínez, A. Geldreich, M. Keller, L.A. Ryabova, TOR and S6K1 promote translation reinitiation of uORF-containing mRNAs via phosphorylation of eIF3h, EMBO J. 32 (2013) 1087-1102.

[23] A.J. Williams, J. Werner-Fraczek, I.F. Chang, J. Bailey-Serres, Regulated phosphorylation of 40S ribosomal protein S6 in root tips of maize, Plant Physiol. 132 (2003) 2086-2097.

[24] H. Reyes de la Cruz, R. Aguilar, E. Sánchez de Jiménez, Functional characterization of a maize ribosomal S6 protein kinase (ZmS6K), a plant ortholog of metazoan p70(S6K), Biochemistry 43 (2004) 533-539.

[25] V. Garrocho-Villegas, C.R. Aguilar, E. Sánchez de Jiménez, Insights into the TOR-S6K signaling pathway in maize (Zea mays L.) pathway activation by effector-receptor interaction, Biochemistry 52 (2013) 9129-9140.

[26] M.A. Block, A.J. Dorne, J. Joyard, R. Douce, Preparation and characterization of membrane fractions enriched in outer and inner envelope membranes from spinach chloroplasts. II. Biochemical characterization, J. Biol. Chem. 258 (1983) $13281-13286$.

[27] A.A. Kelly, J.E. Froehlich, P. Dörmann, Disruption of the two digalactosyldiacylglycerol synthase genes DGD1 and DGD2 in Arabidopsis reveals the existence of an additional enzyme of galactolipid synthesis, Plant Cell 15 (2003) 2694-2706.

[28] K. Kobayashi, M. Kondo, H. Fukuda, M. Nishimura, H. Ohta, Galactolipid synthesis in chloroplast inner envelope is essential for proper thylakoid biogenesis, photosynthesis, and embryogenesis, Proc. Natl. Acad. Sci. U. S. A 104 (2007) 17216-17221.

[29] K. Kobayashi, T. Narise, K. Sonoike, H. Hashimoto, N. Sato, M. Kondo, M. Nishimura, M. Sato, K. Toyooka, K. Sugimoto, H. Wada, T. Masuda, H. Ohta, Role of galactolipid biosynthesis in coordinated development of photosynthetic complexes and thylakoid membranes during chloroplast biogenesis in Arabidopsis, Plant J. 73 (2013) $250-261$. 
[30] K. Düvel, et al, Activation of a metabolic gene regulatory network downstream of mTOR Complex 1, Mol. Cell 39 (2010) 171-183.

[31] T.A. Huffman, I. Mothe-Satney, J.C. Lawrence, Insulin-stimulated phosphorylation of lipin mediated by the mammalian target of rapamycin, Proc. Natl. Acad. Sci. U. S. A. 99 (2002) 1047-1052.

[32] M.K. Holz, B.A. Ballif, S.P. Gygi, J. Blenis, mTOR and S6K1 mediate assembly of the translation preinitiation complex through dynamic protein interchange and ordered phosphorylation events, Cell 123 (2005) 569-580.

[33] M. Laplante, D.M. Sabatini, An emerging role of mTOR in lipid biosynthesis, Cur. Biol. 19 (2009) R1046-R1052.

[34] L.S. Carnevalli, K. Masuda, F. Frigerio, O. Le Bacquer, S.H. Um, V. Gandin, I. Topisirovic, N. Sonenberg, G. Thomas, S.C. Kozma, S6K1 plays a critical role in early adipocyte differentiation, Dev. Cell 18 (2010) 763-774.

[35] S.H. Um, F. Frigerio, M. Watanabe, F. Picard, M. Joaquin, M. Sticker, S. Fumagalli, P.R. Allegrini, S.C. Kozma, J. Auwerx, G. Thomas, Absence of S6K1 protects against age-and diet-induced obesity while enhancing insulin sensitivity, Nature 431 (2004) 200-205.

[36] P. Polak, N. Cybulski, J.N. Feige, J. Auwerx, M.A. Rüegg, M.N. Hall, Adipose-specific knockout of raptor results in lean mice with enhanced mitochondrial respiration, Cell Metab. 8 (2008) 399-410.

[37] T.R. Peterson, S.S. Sengupta,T.E. Harris, A.E. Carmack, S.A. Kang, E. Balderas, D.A. Guertin, K.L. Madden, A.E. Carpenter, B.N. Finck, D.M. Sabatini, .et al, mTOR Complex 1 regulates Lipin 1 localization to control the SREBP pathway, Cell 146 (2011) 408-420.

[38] J. Colombani, S. Raisin, S. Pantalacci, T. Radimerski, J. Montagne, P. Leopold, A nutrient sensor mechanism controls Drosophila growth, Cell 114 (2003) 739-749.

[39] C. Caldana, Y. Li, A. Leisse, Y. Zhang, L. Bartholomaeus, A.R. Fernie, L. Willmitzer, P. Giavalisco, Systemic analysis of inducible target of rapamycin mutants reveal a general metabolic switch controlling growth in Arabidopsis thaliana, Plant J. 73 (2013) 897-909.

[40] Y. Hiei, S. Ohta, T. Komari, T. Kumashiro, Efficient transformation of rice (Oryza sativa L.) mediated by Agrobacterium and sequence analysis of the boundaries of the T-DNA, Plant J. 6 (1994) 271-282.

[41] Z. Wang, C. Benning, Arabidopsis thaliana polar glycerolipid profiling by thin layer 
(TLC) coupled with gas-liquid chromatography (GLC), J. Vis Exp. 49 (2011) 2518.

[42] Y. Hong, X. Pan, R. Welti, X. Wang, Phospholipase D $\alpha 3$ is involved in the hyperosmotic response in Arabidopsis, Plant Cell 20 (2008) 803-816.

[43] Y. Xiong, M. McCormack, L. Li, Q. Hall, C. Xiang, J. Sheen, Glucose-TOR signalling reprograms the transcriptome and activates meristems, Nature 496 (2013) 181-186.

[44] Y.J. Zhang, Y. Duan, X.F. Zheng, Targeting the mTOR kinase domain: the second generation of mTOR inhibitors, Drug Discov. Today 16 (2011) 325-331.

[45] M.H. Montané, B. Menand, ATP-competitive mTOR kinase inhibitors delay plant growth by triggering early differentiation of meristematic cells but no developmental patterning change, J. Exp. Bot. 64 (2013) 4361-4374.

[46] A. Haselier, H. Akbari, A. Weth, W. Baumgartner, M. Frentzen, Two closely related genes of Arabidopsis encode plastidial cytidinediphosphate diacylglycerol synthases essential for photoautotrophic growth, Plant Physiol. 153 (2010) 1372-1384.

[47] G.H. Anderson, B. Veit, M.R. Hanson, The Arabidopsis AtRaptor genes are essential for post-embryonic plant growth, BMC Biol. 3 (2005) 12.

[48] S. Imamura, Y. Kawase, I. Kobayashi, T. Sone, A. Era, S.Y. Miyagishima, M. Shimojima, H. Ohta, K. Tanaka, Target of rapamycin (TOR) plays a critical role in triacylglycerol accumulation in microalgae, Plant Mol. Biol. 89 (2015) 309-318.

[49] Y. Tasaka, Z. Gombos, Y. Nishiyama, P. Mohanty, T. Ohba, K. Ohki, N. Murata, Targeted mutagenesis of acyl-lipid desaturases in Synechocystis: evidence for the important roles of polyunsaturated membrane lipids in growth, respiration and photosynthesis, EMBO J. 15 (1996) 6416-6425.

[50] E.R. Moellering, B. Muthan, C. Benning, Freezing tolerance in plants requires lipid remodeling at the outer chloroplast membrane, Science 330 (2010) 226-228.

[51] C. Bréhélin, F. Kessler, K.J. van Wijk, Plastoglobules: versatile lipoprotein particles in plastids, Trends Plant Sci. 12 (2007) 260-266.

[52] D. Kroll, K. Meierhoff, N. Bechtold, M. Kinoshita,S. Westphal, U.C. Vothknecht, J.

Soll, P. Westhoff, VIPP1, a nuclear gene of Arabidopsis thaliana essential for thylakoid membrane formation, Proc. Natl. Acad. Sci. U. S. A. 98 (2001) 4238-4242.

[53] A. Rudella, G. Friso, J.M. Alonso, J.R. Ecker, K.J. van Wijk, Downregulation of ClpR2 leads to reduced accumulation of the ClpPRS protease complex and defects in chloroplast biogenesis in Arabidopsis, Plant Cell 18 (2006) 1704-1721.

[54] K. Kobayashi, K. Awai, M. Nakamura, A. Nagatani, T. Masuda, H. Ohta, Type-B 
monogalactosyldiacylglycerol synthases are involved in phosphate starvation-induced lipid remodeling, and are crucial for low phosphate adaptation, Plant J. 57 (2009) $322-331$

[55] P. Jarvis, P. Dörmann, C.A. Peto, J. Lutes, C. Benning, J. Chory, Galactolipid deficiency and abnormal chloroplast development in the Arabidopsis MGD synthase 1 mutant, Proc. Natl. Acad. Sci. U. S. A. 97 (2000) 8175-8179.

[56] S. Fujii, K. Kobayashi, Y. Nakamura, H. Wada, Inducible knockdown of MONOGALACTOSYLDIACYLGLYCEROL SYNTHASE1 reveals roles of galactolipids in organelle differentiation in Arabidopsis cotyledons, Plant Physiol. 166 (2014) 1436-1449.

[57] C. Li, Y. Wang, L. Liu, Y. Hu, F. Zhang, S. Mergen, G. Wang, M.R. Schläppi, C. Chu, A rice plastidial nucleotide sugar epimerase is involved in galactolipid biosynthesis and improves photosynthetic efficiency, PLOS Genet. 7 (2011) e1002196.

[58] I. Sakurai, N. Mizusawa, H. Wada, N. Sato, Digalactosyldiacylglycerol is required for stabilization of the oxygen-evolving complex in photosystem II, Plant Physiol. 145 (2007) 1361-1370.

[59] Y. Fang, M. Vilella-Bach, R. Bachmann, A. Flanigan, J. Chen, Phosphatidic acid-mediated mitogenic activation of mTOR signaling, Science 294 (2001) 1942-1945.

[60] T.A. Hornberger, W.K. Chu, Y.W. Mak, J.W. Hsiung, S.A. Huang, S. Chien, The role of phospholipase D and phosphatidic acid in the mechanical activation of mTOR signaling in skeletal muscle, Proc. Natl. Acad. Sci. U. S. A. 103 (2006) 4741-4746.

[61] Y. Hong, S.P. Devaiah, S.C. Bahn, B.N. Thamasandra, M. Li, R. Welti, X. Wang, Phospholipase $\mathrm{D} \varepsilon$ and phosphatidic acid enhance Arabidopsis nitrogen signaling and growth, Plant J. 58 (2009) 376-387.

\section{Figure Legends}

Fig. 1. $S 6 K$ expression pattern, subcellular localization, and activity. (A) $S 6 K 1$ is highly expressed in young and actively growing tissues according to semi-quantitative RT-PCR. R, root; L, leaf; YR, young root; YL, young leaf; St, stem; Sp, spike. The seedling stage, two-week-old seedlings; tillering stage, two-month-old plants; heading stage, three month-old plants. (B) The distinct subcellular localization of rice S6K1 and S6K2, according to the transient expression of GFP-S6K1 and GFP-S6K2 in tobacco epidermal 
cells, observed under laser confocal microscopy. Bars $=30 \mu \mathrm{m}$. (C) Subcellular fractionation of S6K1 by two-phase partitioning. The S6K1-FLAG was expressed in rice plants and detected by Western blotting using anti-FLAG antibody (1:1000). S, soluble fraction; M, microsomal fraction; PM, plasma membrane; IM, intracellular membrane. Protein sample of $20 \mu \mathrm{g} /$ lane was loaded on 10\% SDS-PAGE gel. (D) S6K1 activity assay. The purified S6K1-FLAG was assayed using a synthesized peptide containing the S6 consensus sequence as a substrate. Values are means \pm SD $(n=3)$ from one representative of three independent experiments with similar results. Student's $t$ test, ${ }^{* *} P<0.01$.

Fig. 2. S6K1-suppressed plants exhibited pale yellow-green leaves. (A) The site of T-DNA insertion in the promoter of $S 6 K 1$. Red boxes indicate exons of the coding region, whereas blue boxes indicate the 5'-UTR or 3'-UTR of S6K1. (B) PCR verification of the homozygous $s 6 k$ mutant by PCR products using genomic DNA from WT and $s 6 k 1$ mutant. $\beta$-actin was used as a control. (C) Semi-quantitative RT-PCR of $S 6 K 1$ transcript using RNA from WT and s6k1 mutant. $\beta$-actin was used as an internal control. (D) to (F) Pale yellow-green leaf phenotype of $s 6 k 1$ mutant plants at the different growth stages and the normal green leaves in genetic complementation of the mutant with $S 6 K 1$ driven by its own promoter $(\mathrm{COM})$ or the maize ubiquitin promoter $(\mathrm{OE})$. $(\mathrm{G})$ and $(\mathrm{H})$ Pigment contents $(\mathrm{G})$ and photosynthesis rate $(\mathrm{H})$ in leaves with similar age and position of $\mathrm{WT}$, s6k1 and COM plants at the tillering stage. Values are means $\pm \mathrm{SD}(\mathrm{n}=5)$ from one representative of three independent experiments with similar results. chla, chlorophyll a; chlb, chlorophyll b; car, carotenoid. (I) Starch content of leaves with similar age and position of WT, s6k1 and COM plants was tested at the tillering stage. Values are means $\pm \operatorname{SD}(n=5)$ from one representative of three independent experiments with similar results. (J) Thousand-grain weight was investigated after the seeds were collected from mature plants grown in the field. Values are means \pm SD $(n=16)$ from one representative of three independent experiments with similar results. Student's $t$ test, ** $P<0.01$.

Fig. 3. The effect of S6K1 on thylakoid grana architecture. (A) (B) Transverse sections of leaves during the seedling (A) and tillering stage (B) were observed under TEM. The black arrows indicate thylakoid grana, white arrows indicate plastoglobules, and white arrowheads indicate starch granules. (C) Choloroplast size in WT and $s 6 k 1$ plants, Values are means \pm SD $(n=3)$ from three independent experiments. Data was measured from 20 
cells in each experiment. (D) The amounts of plastoglobules within chloroplasts between $s 6 k 1$ and WT plants. Values are means \pm SD $(n=3)$ from three independent experiments. Data was collected from 20 cells in each experiment. Student's $t$ test, $* * P<0.01$.

Fig. 4. The effect of S6K1 on galactolipid contents and fatty acid composition. (A) Contents of total polar lipids extracted from leaves of WT, $s 6 k 1$, and COM plants at the seedling stage. (B) and (C) The contents of fatty acid species of galactolipid MGDG (B) and DGDG (C) in leaves at the seedling stage. Fatty acid species were shown as total acyl carbons : total double bonds. Values are means \pm SD $(n=3)$ from one representative of three independent experiments with similar results. Student's $t$ test, $* P<0.05$, $* * P<0.01$.

Fig. 5. S6K1 interacts with Raptor2. (A) Immunoblotting of S6K1 and Raptor co-immunoprecipitated from rice leaves. Proteins from transgenic plants carrying S6K1-FLAG were immunoprecipitated using an anti-FLAG antibody attached to affinity M2 gel and probed with an anti-Raptor antibody. (B) Immunoblotting of S6K1 and Raptor2 co-pull down using recombinant S6K1-GST. Raptor2-His incubated with immobilized GST or the GST-fused S6K1 was pulled down by glutathione agarose affinity gel and probed with an anti-His antibody. (C) S6K1-FLAG incubated with Raptor2-His was precipitated using Ni-NTA His bind resin and probed with an anti-FLAG antibody. The proteins from cells containing the pET28a(+) vector only were used as a control.

Fig. 6. Loss of Raptor 2 resulted in pale leaves and defective thylakoid grana architecture. (A) The location of T-DNA insertion in Raptor2. Red boxes indicate exons of Raptor2. (B) Loss of Raptor 2 transcript in the raptor 2 mutant by semi-quantitative RT-PCR. $\beta$-actin was used as an internal control. (C) and (D) The Raptor2 deficient plants exhibited striped pale or white leaf phenotype. The pictures were taken from plants at the tillering stage. Striped pale, leaf colors with alternate stripes of green and white; white, leaf colors with completely white. (E) and (F) Suppressed Raptor2 transcript by RNA interference (RNAi) also caused a white leaf phenotype. Values are means \pm SD ( $n=3$ separate samples). (G) Pigment contents in leaves of raptor 2 and WT plants at the tillering stage. Values are means \pm SD (n = 3) from one representative of three independent experiments with similar results. $(\mathrm{H})-(\mathrm{J})$ Defective thylakoid grana architecture in raptor 2 leaves. The pictures were taken from transverse sections of leaves at the tillering stage. The black arrows indicate thylakoid 
grana, white arrows indicate plastoglobules, and white arrowheads indicate starch granules. (K) The effect of Raptor2 on S6K phosphorylation. Phosphorylated S6K in leaves of seedlings was detected using anti-phospho-p70S6K (pThr389) antibodies. (L) The effect of Raptor2 on S6K1 transcript. Total RNA was extracted from leaves of raptor2 and WT plants at the seedling stage and was quantified by real-time PCR normalized to $\beta$-actin. Values are means \pm SD ( $n=3$ separate samples). (M) The effect of Raptor2 on S6K activity. The same amounts of proteins $(10 \mu \mathrm{g})$ extracted from the leaves of pale raptor 2 and WT plants at the seedling stage were used for S6K1 activity assay using a synthesized peptide containing the S6 consensus sequence (KRRRLASLR) as the substrate. Values are means \pm $\mathrm{SD}(\mathrm{n}=3)$ from one representative of three independent experiments with similar results. (N) The effect of Raptor2 on thylakoid membrane biosynthesis. Total polar lipids were extracted from leaves of raptor 2 and WT plants at the seedling stage. The resultant lipids were separated on a TLC plate and were quantitatively analyzed by GC-MS. Values are means \pm SD $(n=3)$ from one representative of three independent experiments with similar results. Student's $t$ test, $* * P<0.01$.

Fig. 7. Suppressed TOR activity by Torin 2 and AZD8055 conferred pale yellow-green leaves and lipid alteration. (A) and (B) Two-week-old seedlings were treated with different concentrations $(5,30 \mu \mathrm{M})$ of Torin2 (A) and AZD8055 (B) for 5 days. (C) TOR activity indicated by phosphorylated S6K was suppressed by TOR inhibitors Torin2 (30 $\mu \mathrm{M})$ and AZD8055 $(30 \mu \mathrm{M})$ in different time intervals. Phosphorylated S6K in leaves of seedlings was detected using anti-phospho-p70S6K (pThr389) antibodies. (D) Pigment contents of leaves from seedlings shown in (A). Values are means $\pm \operatorname{SD}(n=6)$ from one representative of three independent experiments with similar results. (E) Suppressed TOR activity by Torin2 or AZD8055 resulted in significantly reduced galactolipids. Lipids were extracted from leaves of seedlings grown in (A) conditions for 5 days. The resultant lipids were separated by TLC and were quantitatively analyzed by GC measurement. Values are means \pm SD $(n=3)$ from one representative of three independent experiments with similar results. Student's $t$ test, $* * P<0.01$.

Fig. 8. The transcriptional profiling of lipid-related genes. (A), (B) and (D) Transcript levels of lipid-related genes in WT, s6k1, raptor2, and $c d s 5$ plants. (C) The expression levels of lipid-related genes in response to Torin2 and AZD8055 treatments. Total RNA 
931

932

933

934

935

936

937

938

939

940

941

942

943

944

945 was extracted from leaves of the seedlings treated with Torin2 $(30 \mu \mathrm{M})$ and AZD8055 (30 $\mu \mathrm{M})$, respectively, for 24 hours. The expression level was profiled by quantitative real-time PCR normalized to $\beta$-actin. Values are means $\pm \mathrm{SD}(\mathrm{n}=3$ separate samples). $M G D$, MGDG synthase; DGD, DGDG synthase; LPPa, lipid phosphate phosphatase $\alpha$; NPC, non-specific phospholipase C; PLD $\alpha 1$, phospholipase D $\alpha 1$; CDS5, cytidinediphosphate diacylglycerol synthase 5. (E) and (F) Loss of LPP $\alpha 5$ led to pale yellow-green leaves. (G) Pigment contents in leaves of lpp $\alpha 5$ and WT plants at the tillering stage. Values are means $\pm \mathrm{SD}(\mathrm{n}=3)$ from one representative of three independent experiments with similar results. Student's $t$ test, $* P<0.05$, ** $P<0.01$.

Fig. 9. A work model for TOR-Raptor2-S6K1 in galactolipid biosynthesis. TOR, Raptor2, and S6K1 coordinately regulate the transcript levels of genes encoding LPP $\alpha 5$, MGD1, and DGD1 involved in galactolipid synthesis to modulate thylakoid grana architecture for normal photosynthesis in rice. 
A

S6K1

S6K2

$\beta$-actin

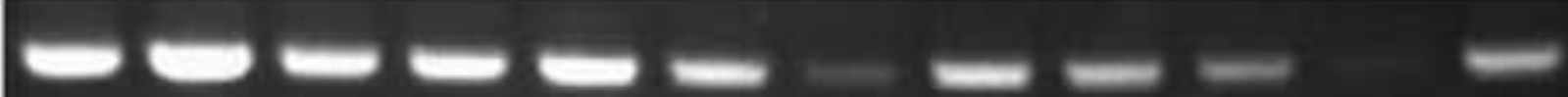

\section{A}

(1)

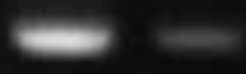
(5) $\frac{\mathrm{R} \quad \mathrm{L}}{\text { Seedling }} \frac{\mathrm{YR} \quad \mathrm{R} \quad \mathrm{YL} L}{\text { Tillering stage }} \quad \frac{}{\text { Heading stage }}$

B

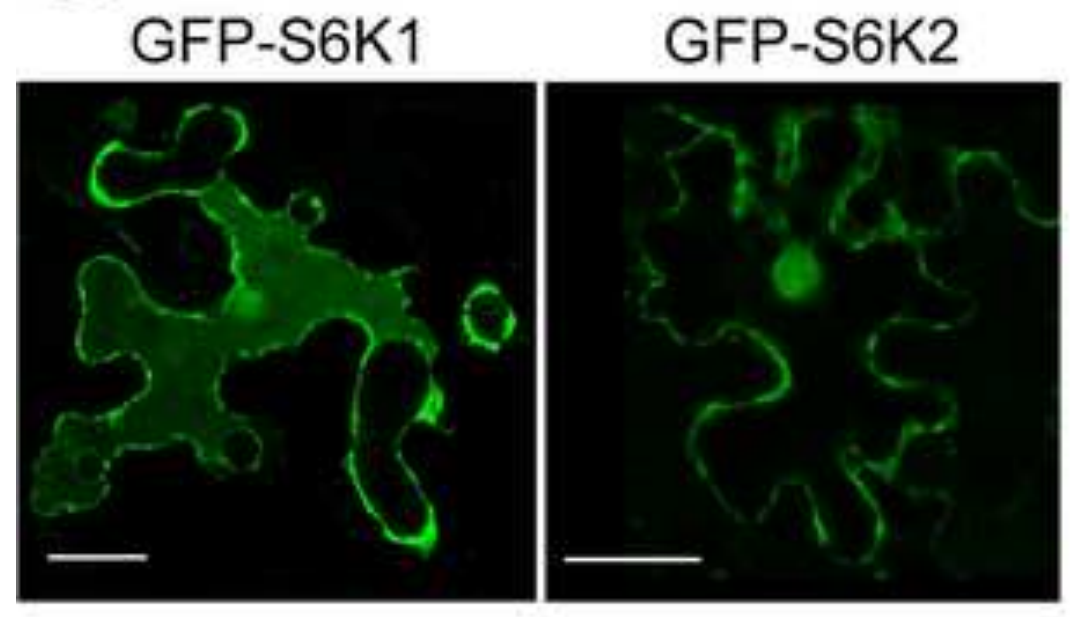

C S6K1
D

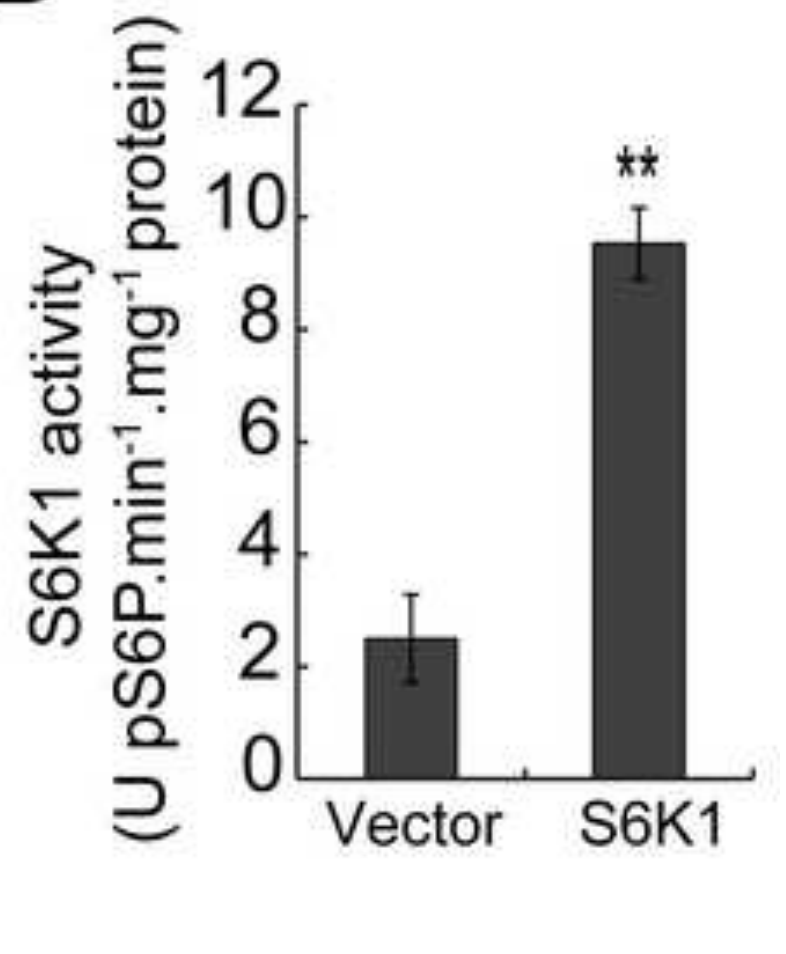

\section{$\begin{array}{llll}S & M & P M & \text { IM }\end{array}$}


A

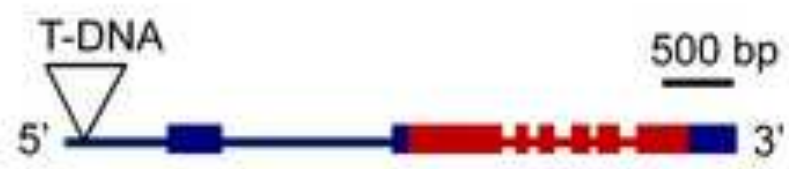

D

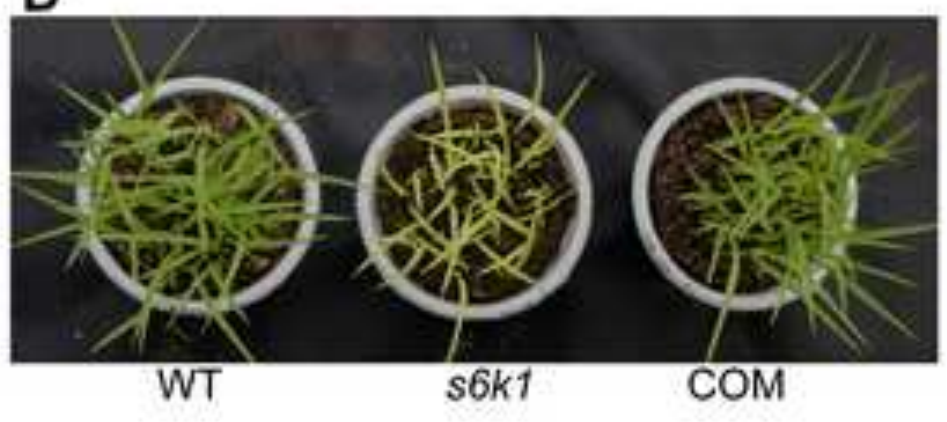

E
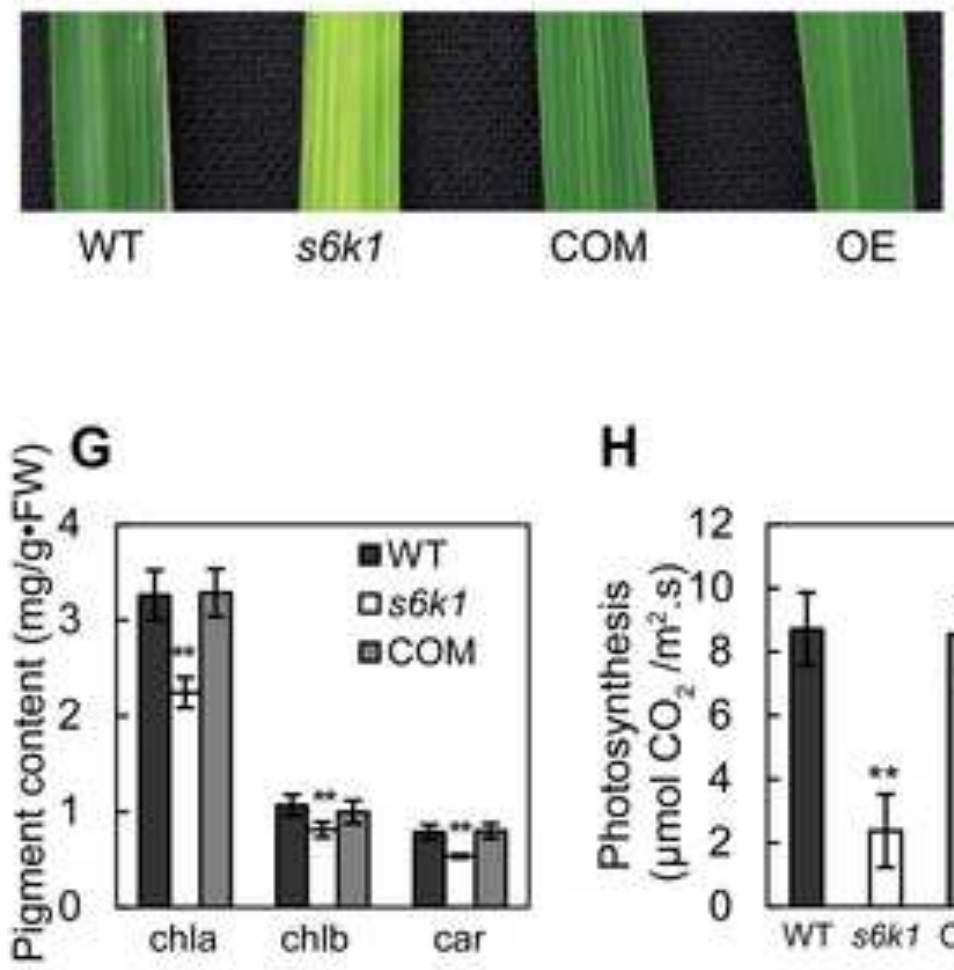

H

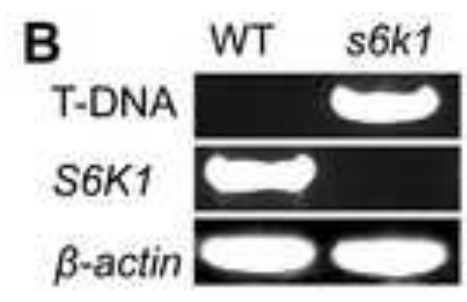

F
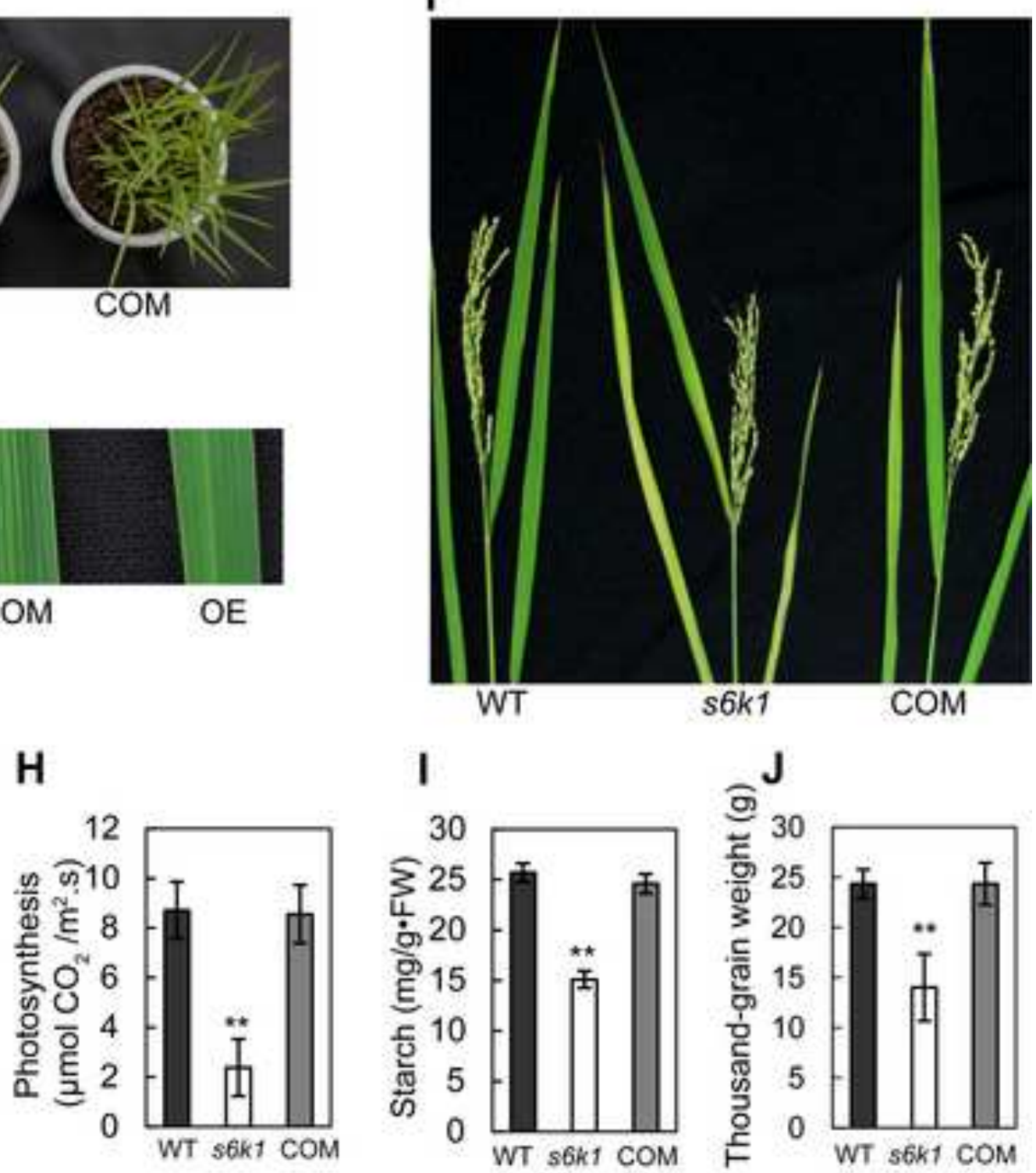

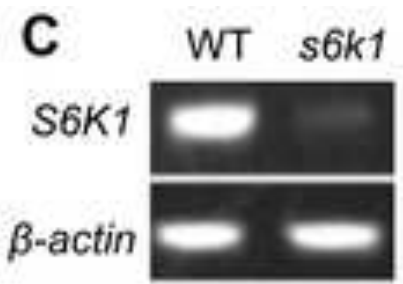

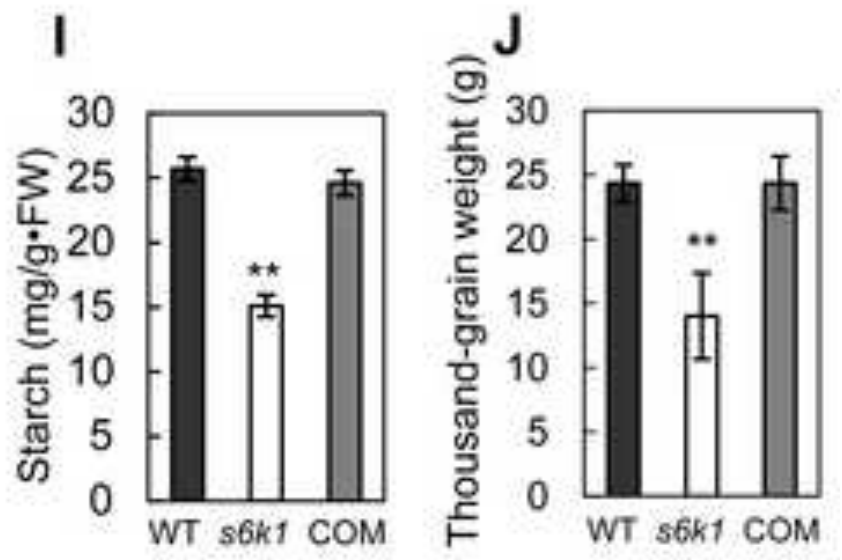


A

Seedling stage

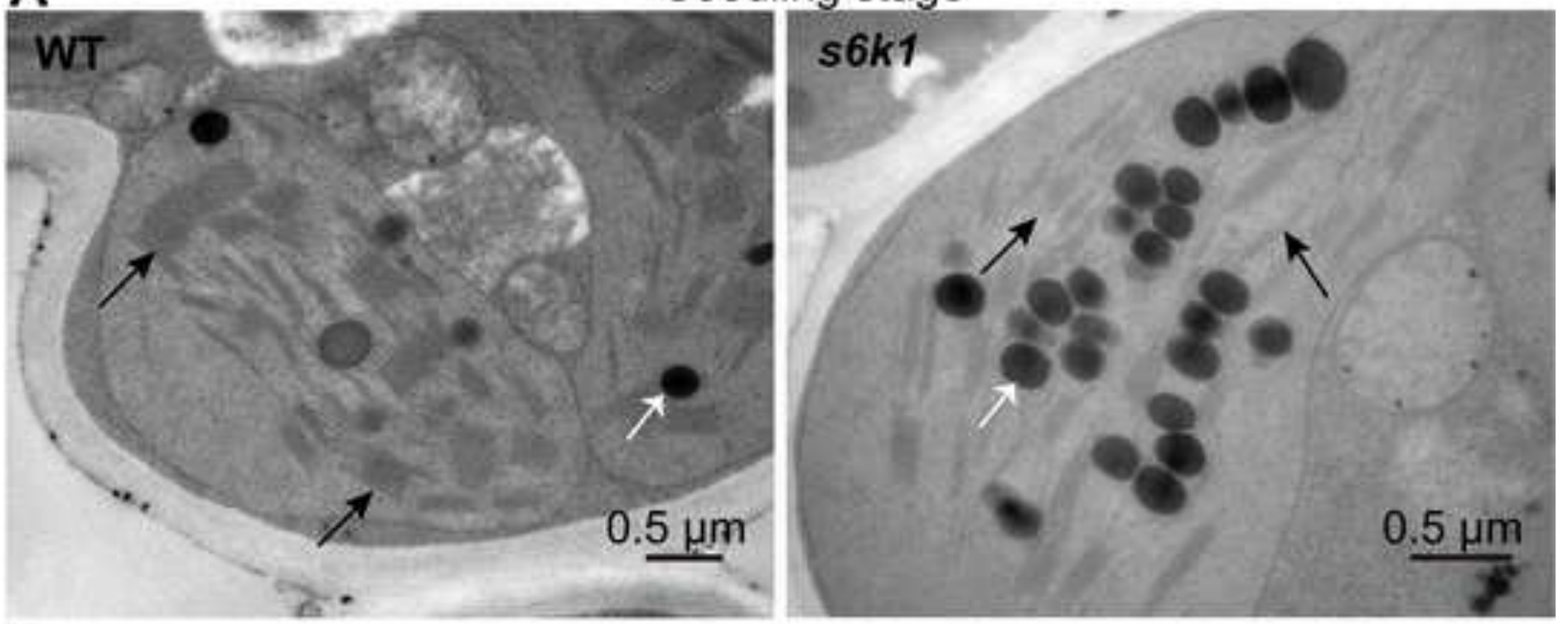

B

Tillering stage
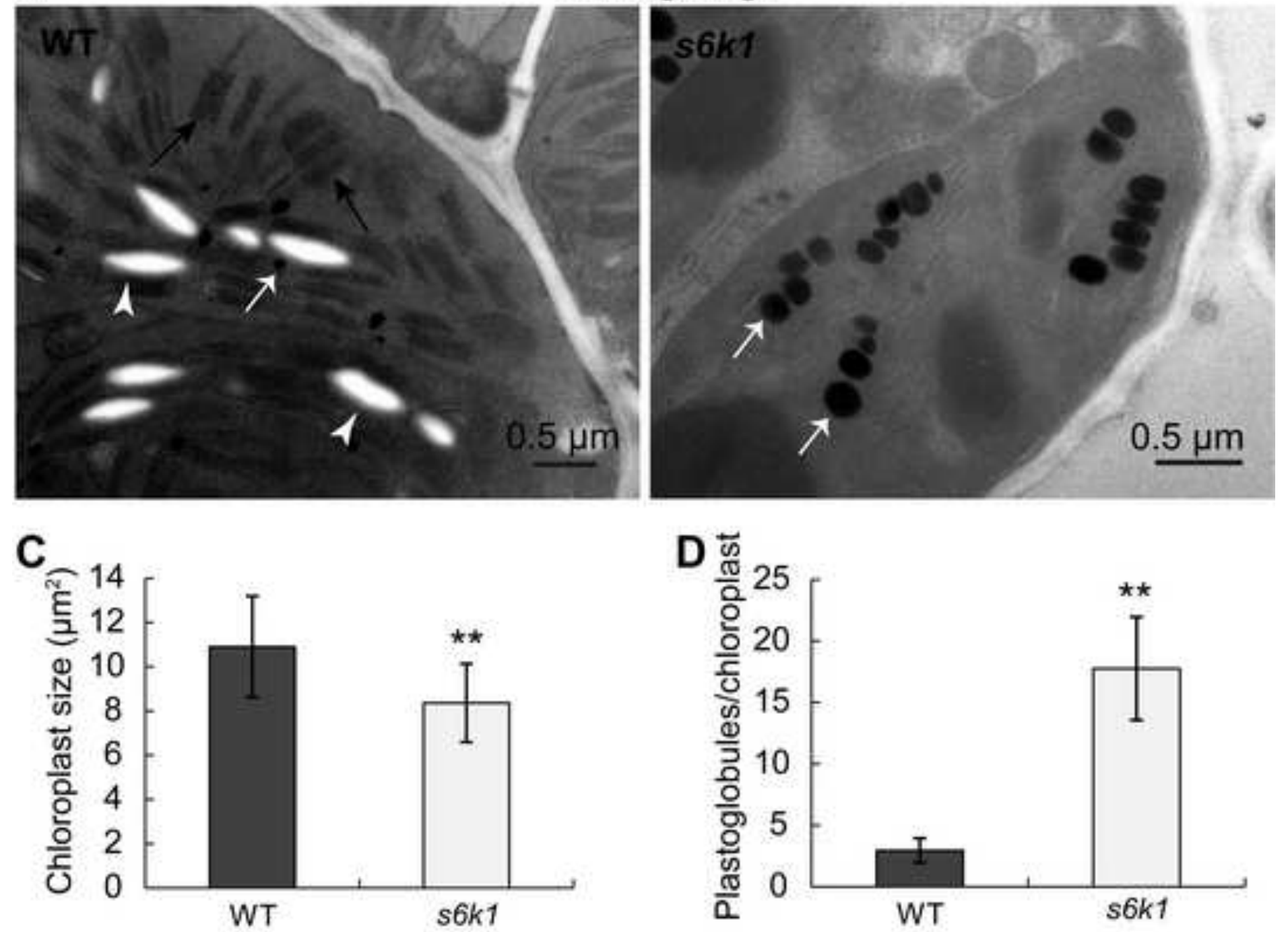

D

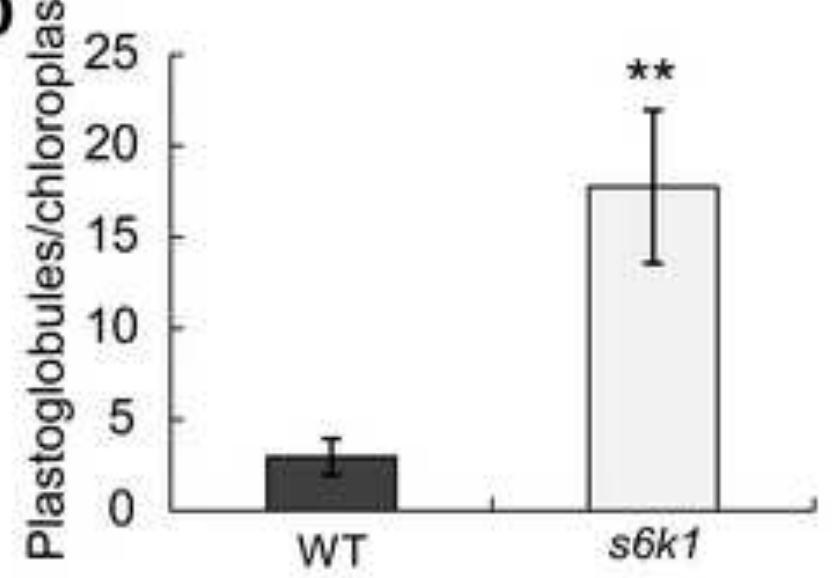




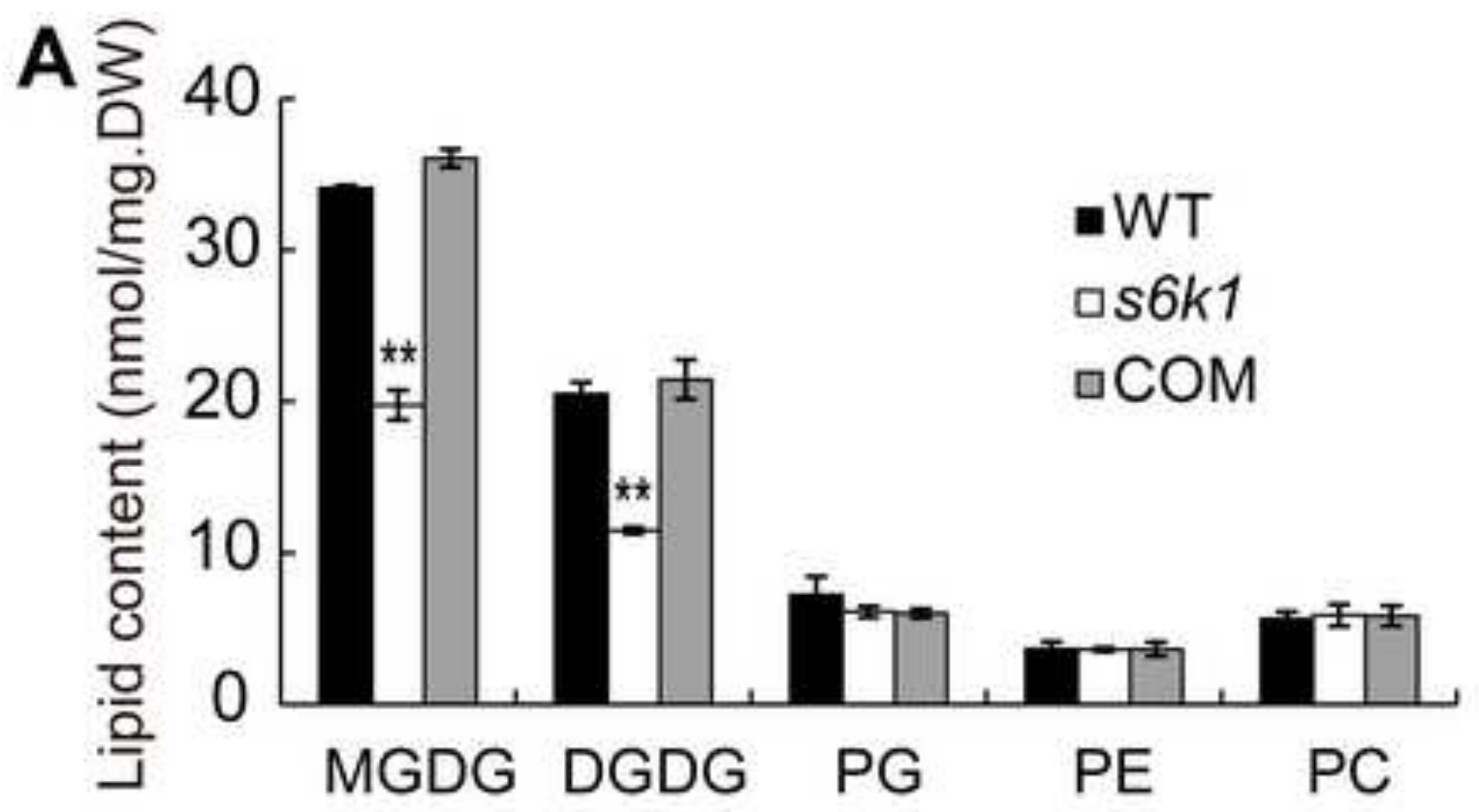

\section{B}

동 50 [

过 50

흥 흥 40 .

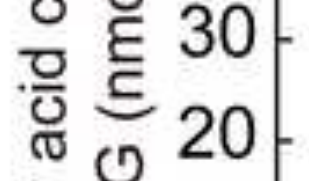

密㒭 10

$\begin{array}{llllll}16: 0 & 16: 1 & 18: 0 & 18: 1 & 18: 3 & 20: 1\end{array}$

C
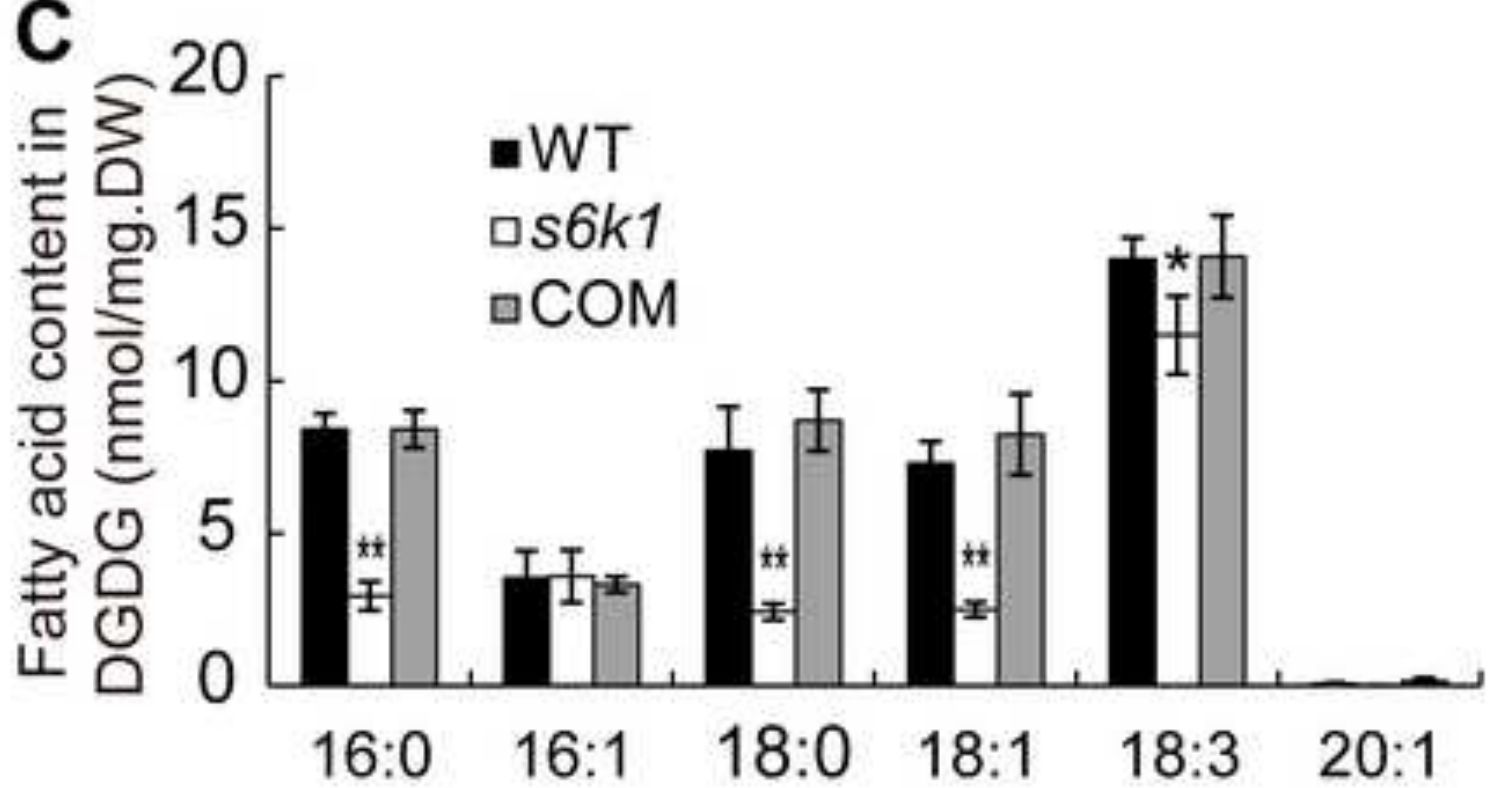
A
S6K1-FLAG
Raptor
Anti-FLAG

Anti-Raptor
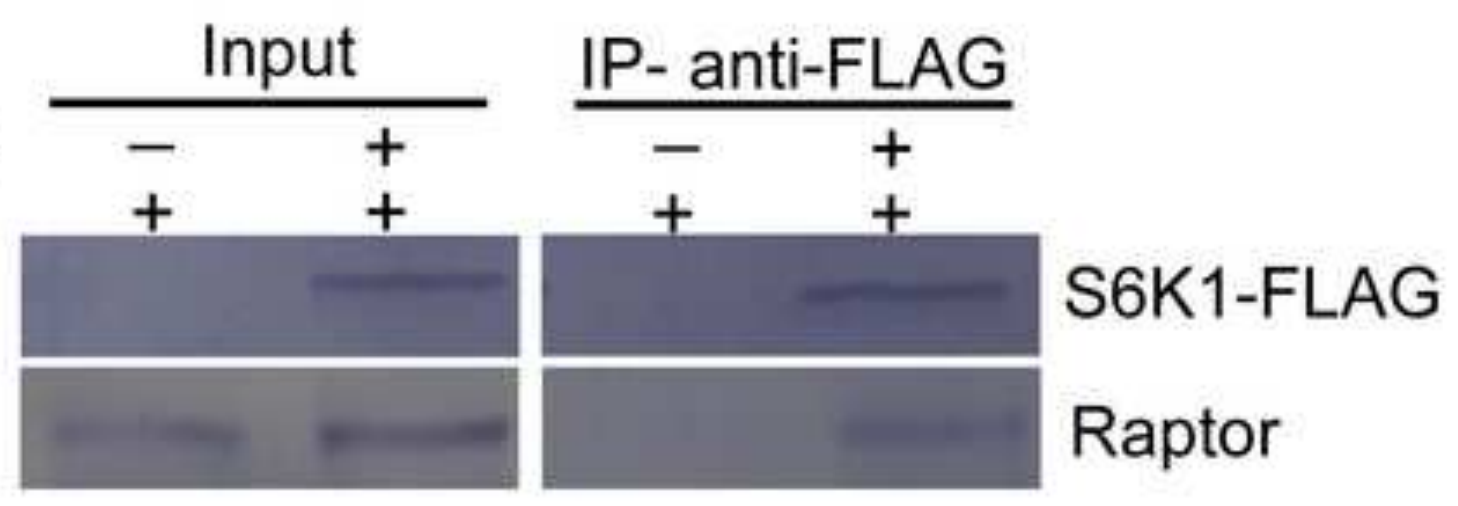

B

S6K1-GST

GST

Raptor2-His

Anti-GST

Anti-His

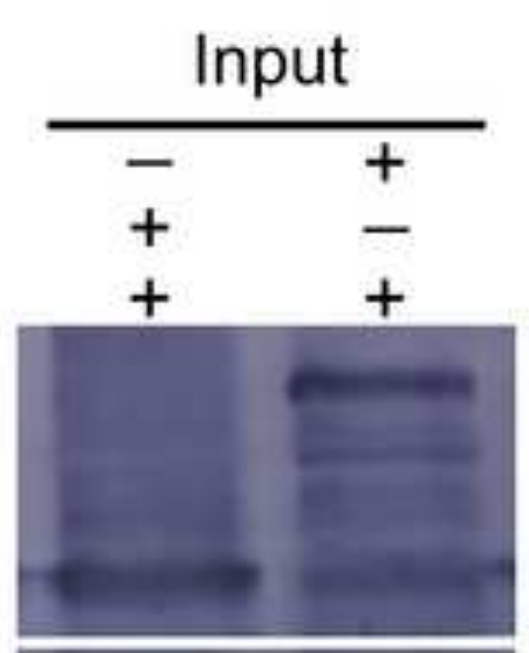

GST pulldown

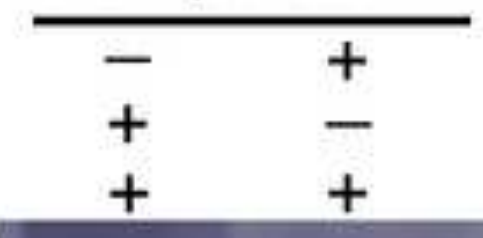

S6K1-GST

\section{GST}

Raptor2-His
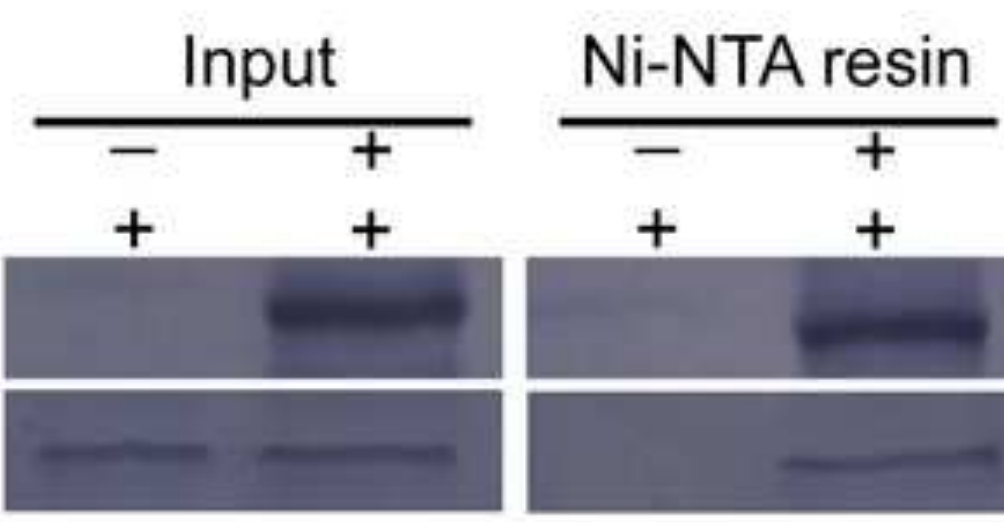
Anti-His Anti-FLAG

Raptor2-His S6K1-FLAG 

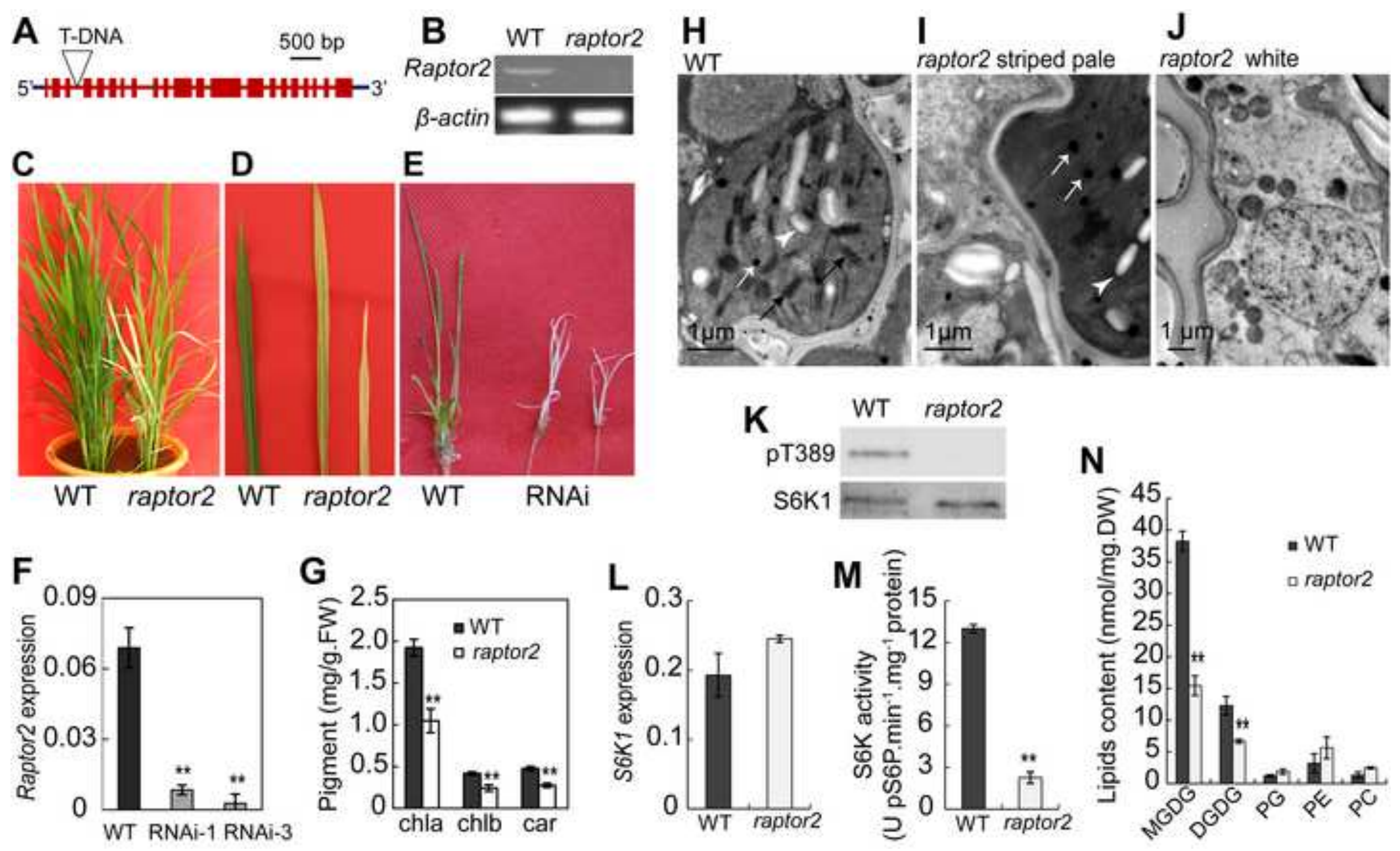
A

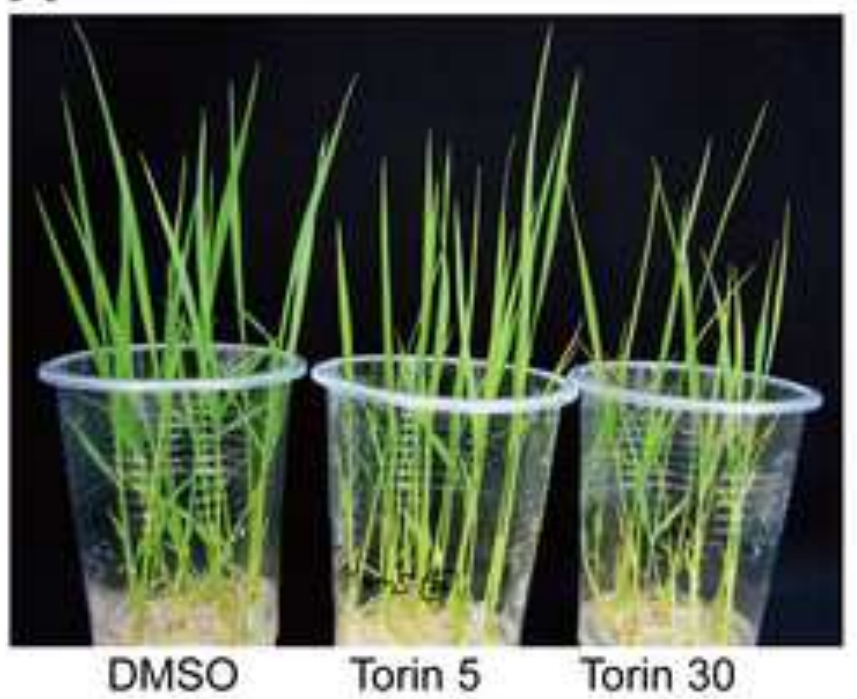

B

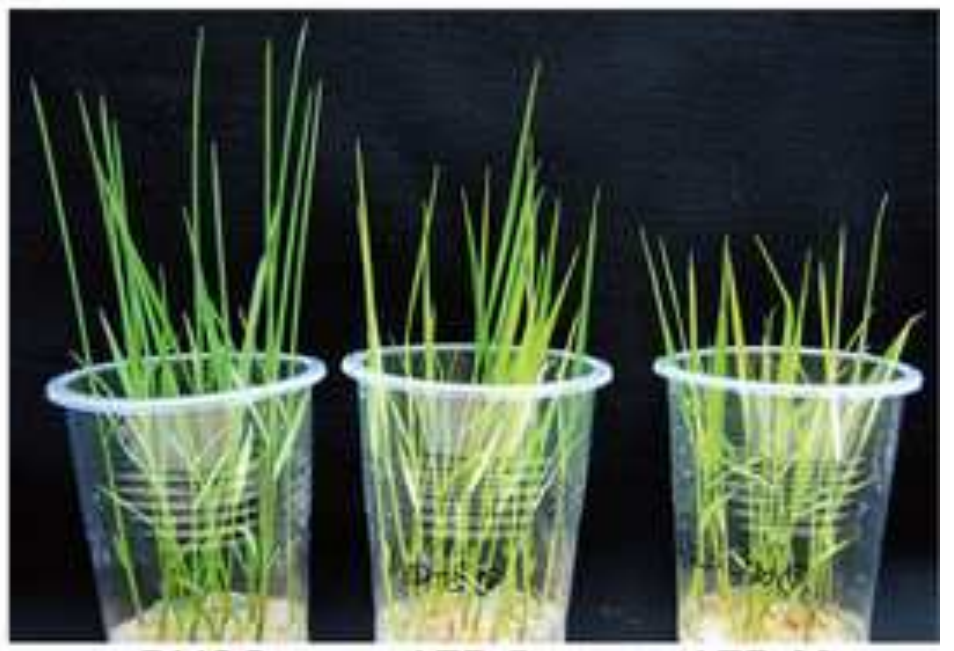

DMSO

AZD 5

AZD 30

C

рT389

S6K1

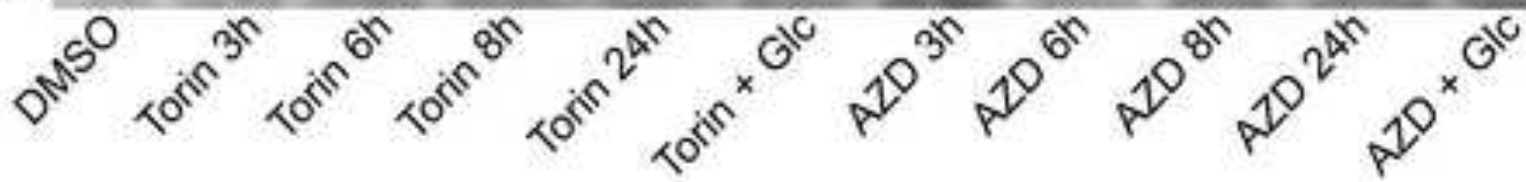

D

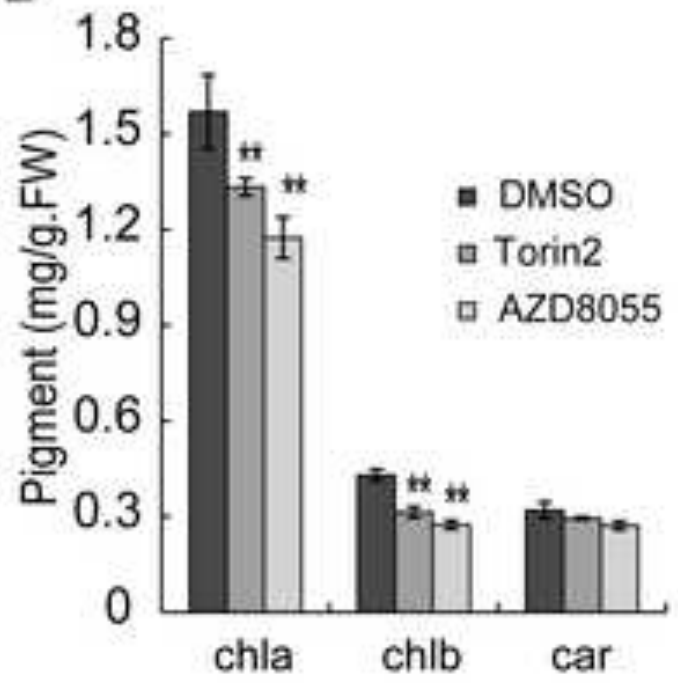

E

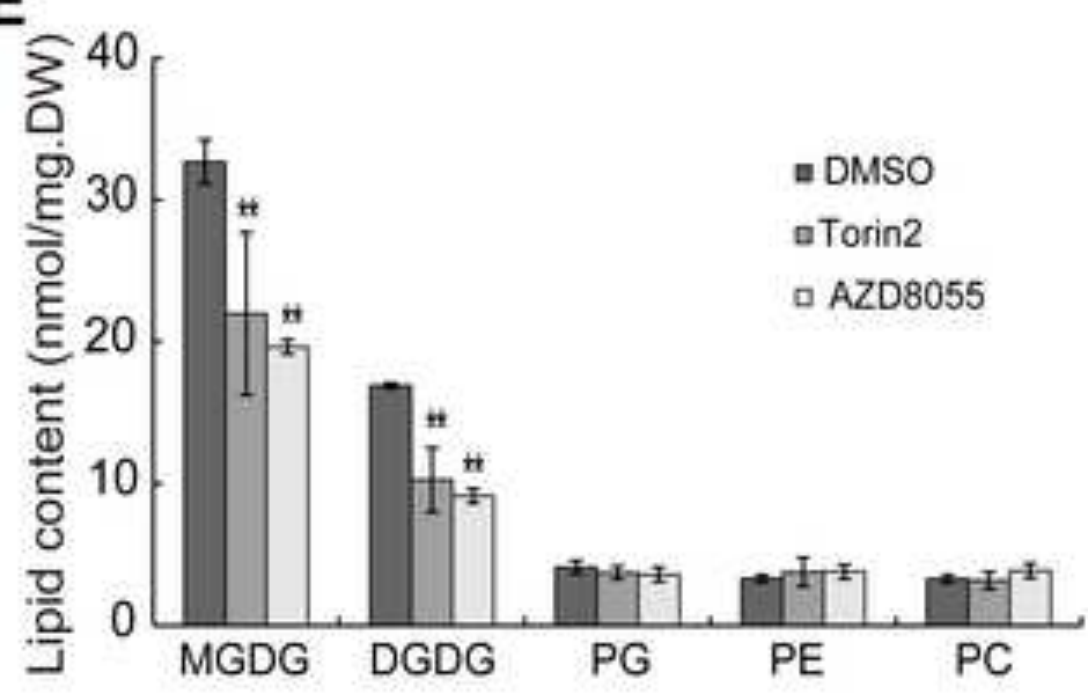




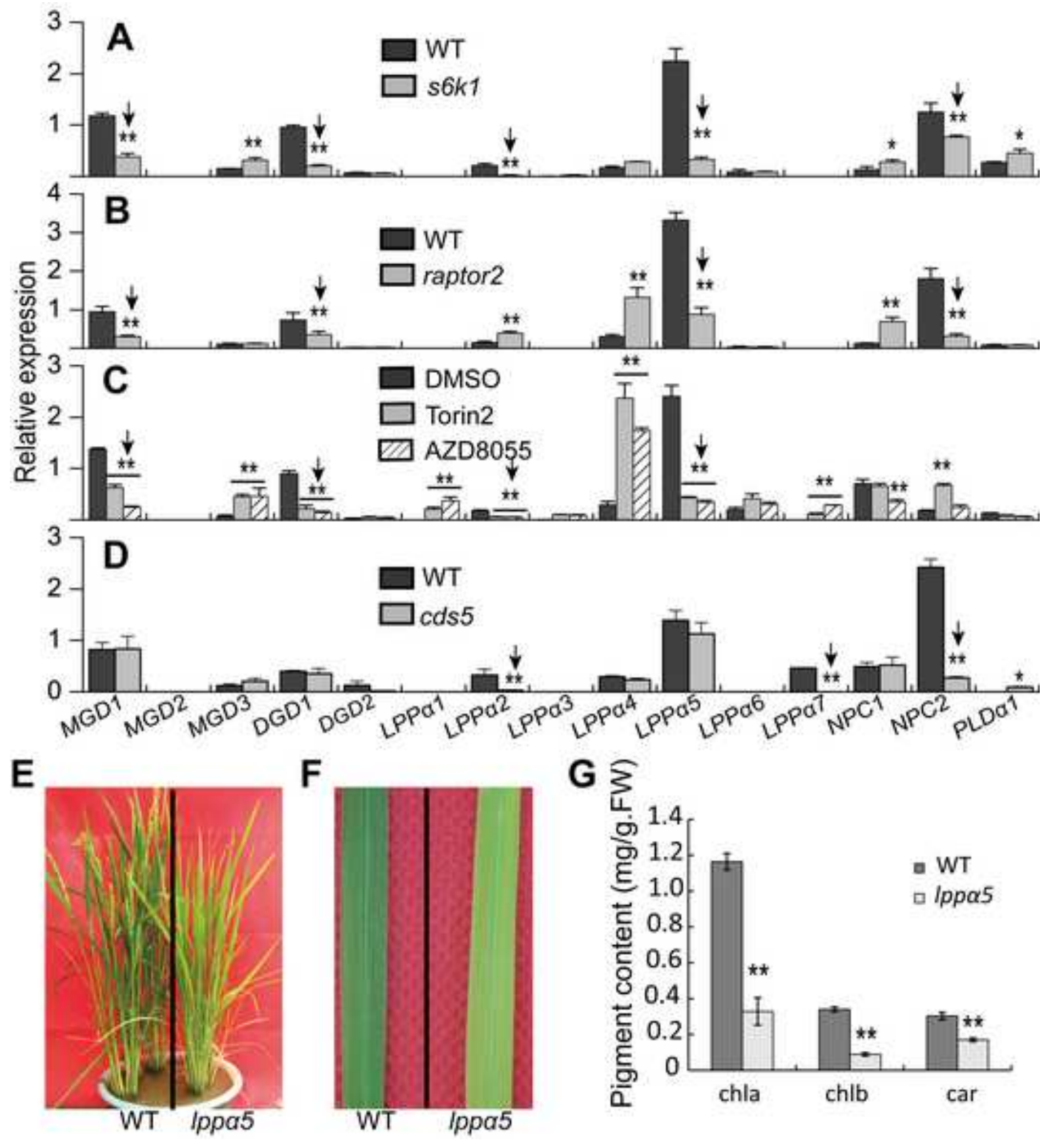


